\title{
Recognition of high-risk HPV E6 oncoproteins by 14-3-3 proteins studied by interactomics and crystallography
}

\section{Gergő Gógl}

University of Strasbourg

\section{Kristina Tugaeva}

A N Bach Institute of Biochemistry

\section{Pascal Eberling}

Department of Integrated Structural Biology, Institut de Genetique et de Biologie Moleculaire et Cellulaire (IGBMC)

\section{Camille Kostmann}

University of Strasbourg

Gilles Trave

Institute of Genetics and Molecular and Cellular Biology

Nikolai Sluchanko ( $\nabla$ nikolai.sluchanko@mail.ru )

A N Bach Institute of Biochemistry https://orcid.org/0000-0002-8608-1416

\section{Article}

Keywords: Protein chimera, protein-protein interactions, phosphopeptides, phosphorylation, crystal structure, binding affinity

Posted Date: October 26th, 2020

DOI: https://doi.org/10.21203/rs.3.rs-48671/v1

License: (c) (1) This work is licensed under a Creative Commons Attribution 4.0 International License. Read Full License

Version of Record: A version of this preprint was published at Nature Communications on March 15th, 2021. See the published version at https://doi.org/10.1038/s41467-021-21908-8. 


\section{Recognition of high-risk HPV E6 oncoproteins by 14-3-3 proteins studied by}

interactomics and crystallography

4 Gergo Gogl ${ }^{1 *}$, Kristina V. Tugaeva ${ }^{2 *}$, Pascal Eberling ${ }^{1}$, Camille Kostmann ${ }^{1}$, Gilles Trave ${ }^{1 \dagger}$, 5 Nikolai N. Sluchanko ${ }^{2 \dagger}$

71 Equipe Labellisee Ligue 2015, Department of Integrated Structural Biology, Institut de 8 Genetique et de Biologie Moleculaire et Cellulaire (IGBMC), INSERM U1258/CNRS UMR 9 7104/Universite de Strasbourg, 1 rue Laurent Fries, BP 10142, F-67404 Illkirch, France

102 A.N. Bach Institute of Biochemistry, Federal Research Center of Biotechnology of the 11 Russian Academy of Sciences, 119071, Moscow, Russia

12

13 Email addresses of the authors:

14 goglg@igbmc.fr

15 kri9408@mail.ru

16 eberling@igbmc.fr

17 kostmanc@igbmc.fr

18

$19{ }^{*}$ Contributed equally: G.G. and K.V.T.

20 †Corresponding authors: G.T.: traveg@igbmc.fr ; N.N.S.: nikolai.sluchanko@mail.ru 
In tumors induced by high-risk mucosal human papillomaviruses (hrm-HPVs), HPV E6 oncoproteins inhibit apoptotic processes and sustain cell proliferation. E6 from all hrmHPVs harbor a C-terminal short PDZ domain-binding motif (PBM), whose phosphorylation down-regulates PDZ binding but triggers E6 binding to 14-3-3 proteins. Here we classify PBMs of E6 proteins depending on their principle ability to be phosphorylated and subsequently acquire a 14-3-3-binding motif III consensus, (pS/pT)XX-COOH. Systematic competitive fluorescence polarization measurements show that the PBMs from four selected E6 oncoproteins bind all seven human 14-3-3 isoforms with distinct, wide-ranging affinities, obeying remarkable trends assigned to 14-3-3 isoform specificity and small E6 sequence variations. We crystallized the hrm-HPV18 E6 PBM bound to 14-3-3弓, revealing a 14-3-3-motif III complex at $1.9 \AA$ resolution. Using fluorescence polarization and crystallography, we also demonstrate that fusicoccin, a molecule that reinforces many known 14-3-3 complexes, destabilizes the 14-3-3-E6 interaction, indicating the druggability of that complex.

Keywords:

Protein chimera; protein-protein interactions; phosphopeptides; phosphorylation; crystal structure; binding affinity

\section{INTRODUCTION}

Papillomaviruses (PVs) are a large group of small oncogenic DNA viruses infecting various vertebrates including humans (human papillomaviruses, HPVs). PVs target mucosal or cutaneous epithelium cells and induce their proliferation, to amplify their circular doublestranded DNA genome and package it into virion particles ready for a new infectious cycle. 225 HPV types currently annotated in the Papillomavirus Episteme (PaVE) database (https://pave.niaid.nih.gov/) are classified into five phylogenetically distinct genera (alpha, beta, gamma, mu and nu), of which alpha HPVs display almost exclusively mucosal tropism ${ }^{1}$. While most HPV only generate benign proliferative events such as warts, lesions or condylomas, a subset of about 20 HPV types belonging to two alpha species have been associated to cancers and are therefore epidemiologically categorized as "high-risk" 2, 3, 4 . HPV16 and 18 are the most prevalent, causing up to $80 \%$ of squamous cervical carcinomas ${ }^{5}$, whereas HPV16 is also highly prevalent in HPV-positive tumors of the oropharynx and the anus ${ }^{6}$.

The E6 protein is one of the two main HPV oncoproteins expressed at the early stage of infection. In HPV-transformed cells, E6 participates in counteracting apoptosis, altering differentiation pathways, polarity and adhesion properties, and thereby sustains cell proliferation ${ }^{1,7}$. Inhibition of E6 in HPV-positive cell lines results in the cell growth arrest and induces apoptosis or rapid senescence ${ }^{8,9,10,11 .}$

E6 has been found to interact with numerous distinct cellular target proteins involved in a variety of cellular functions ${ }^{12}$. Importantly, E6 proteins from distinct HPV species recognize distinct subsets of the full panel of potential E6 targets ${ }^{13}$. This likely contributes to the 63 particular biological traits of each HPV type in terms of tropism, viral cycle, or 64 pathogenicity. 
E6 proteins of most mammalian PVs comprise two zinc-binding domains (E6N and E6C) separated by a linker helix ${ }^{1}$ (Fig. 1A), which altogether form a charged-hydrophobic pocket that recognizes $L x x L L$ motifs interspaced with acidic residues, found in a variety of host cellular proteins ${ }^{12}$. Hrm-HPV E6 recruits simultaneously the LxxLL motif of ubiquitin ligase E6-Associated Protein (E6AP) and, via a distinct interface, the central "core" domain of tumor suppressor p53 ${ }^{14}$. The resulting E6-E6AP-p53 trimer drives the ubiquitinylation and subsequent proteasomal degradation of $p 53^{15}$. In HPV-transformed cells, this leads to low levels of p53 protein that probably contribute to the tumorigenic phenotype. It is supposed that E6 similarly stimulates the E6AP-mediated degradation of other host proteins ${ }^{15}, 16$.

In addition to their LxxLL-binding pocket and p53-binding surface, hrm-HPV E6 proteins harbor a C-terminal PDZ domain-binding motif (PBM) ${ }^{17}$ (Fig. 1A), which remarkably is absent in most low-risk non-cancer-causing HPV E6 proteins ${ }^{18}$. The intrinsically disordered PBM enables the E6 interaction with a variety of host PDZ domain-containing proteins involved in the regulation of cell polarity, adhesion, differentiation or survival ${ }^{19}$. These interactions of E6 with PDZ proteins are potentially modulated by PBM phosphorylation. Several E6 PBMs are phosphorylatable in vitro at variable levels of specificity and efficiency by basophilic kinases, such as PKA ${ }^{18,20,21}$, PKB/Akt ${ }^{18,21}$, CamKII ${ }^{21}$, or Chk1, and by the glutamine-directed ATM/ATR kinases ${ }^{22}$. E6 PBMs from high-risk mucosal HPV types 16, 18, 33, 35 (Fig. 1B) are good substrates for PKA in vitro ${ }^{18,21,23}$. In addition, E6 of HPV16 and HPV18 (hereinafter called 16E6 and 18E6) are metabolically labeled by ${ }^{32} \mathrm{P}$ upon transient transfection in vivo, and forskolin promotes such labeling ${ }^{23}$. Phosphorylation of several HPV-E6 proteins at the PBM was confirmed by using phosphospecific antibodies raised against the C-terminal PBM of $18 \mathrm{E} 6{ }^{18,21}$. It was also shown that the level of $18 \mathrm{E} 6$ phosphorylation remains low in the normal cell cycle, until the activation of stress-response kinases and those involved in the DNA damage response (see in particular ${ }^{22}$ ).

91 While phosphorylation of HPV-E6 PBMs generally reduces their binding to PDZ domains

$92{ }^{24}$, it turns several of them into candidate binding sites for $14-3-3$ proteins ${ }^{18,21}$. Despite being encoded by seven distinct genes, human 14-3-3 proteins share highly similar sequences and biochemical properties. Hence they are commonly referred to as "isoforms", individually named $\beta, \gamma, \varepsilon, \zeta, \eta, \sigma$, and т (beta, gamma, epsilon, zeta, eta, sigma and tau ${ }^{25}$, not to be confused with their splice variants. 14-3-3 proteins form homo- and heterodimers ${ }^{26}$ characterized by their ability to bind phosphopeptides ${ }^{27,} 28$. 14-3-3 are involved in the regulation of multiple cellular processes including apoptosis, cell division and signal transduction ${ }^{29}$. Phosphorylated 14-3-3-binding sequences usually correspond to internal motifs I RSX(pS/pT)X(P/G) and II RXY/FX(pS/pT)X(P/G) ${ }^{28}$ and to the C-terminal

101 motif III (pS/pT) $\mathrm{X}_{0-2}-\mathrm{COOH}^{30,31}$, where $\mathrm{pS} / \mathrm{pT}$ denotes phosphorylated serine or threonine

103

104

105 targets from dephosphorylation and degradation, affects their activity, intracellular localization, and interactions with other proteins by occlusion of the phosphorylated segments ${ }^{32}$. Given the involvement of $14-3-3$ in the development of various pathological

106 conditions associated with the deregulation of the corresponding protein-protein 107 interactions, 14-3-3/phosphotarget complexes are considered with increasing attention ${ }^{33}$.

108 Phosphorylated 16E6, 18E6, and 31E6 have been previously shown to bind to 14-3-3 109 proteins, albeit with different efficiencies 18, 21, 24 . In contrast, the binding of 110 unphosphorylated or phosphomimetic E6 PBMs to 14-3-3 proteins was not detectable ${ }^{21,24}$. 
111 This suggests that the phosphorylation-dependent interaction with 14-3-3 proteins is a 112 general feature of various E6 PBMs.

113 Here we classified PBMs of hrm-HPV E6 proteins depending on their principle ability to be 114 phosphorylated and subsequently acquire a 14-3-3-binding motif III consensus, (pS/pT)XX$115 \mathrm{COOH}$. We performed systematic quantitative binding assays of four phosphorylatable E6 116 PBMs with all seven human 14-3-3 isoform homodimers and solved a high-resolution 117 crystal structure of the HPV18 E6 PBM complex with 14-3-3ろ. This combination of 118 approaches has allowed us to rationalize differences in the affinities of tested PBMs and 119 fully confirm this by mutated peptides. Finally, we used binding assays and crystallography 120 to analyze the impact of natural toxin fusicoccin on the 14-3-3-E6 PBM interaction, which 121 proved that the 14-3-3-E6 complexes are nominally druggable by small molecules.

\section{RESULTS}

123 PDZ-binding motifs of E6 proteins can be classified according to their phosphorylation and 14-3-3-binding propensity

Among all 225 HPV type E6 proteins curated in the PaVE database (https://pave.niaid.nih.gov/), 31 E6 proteins from the a-genera HPV, having predominantly mucosal tropism, possess a C-terminal PDZ-binding motif (PBM) that corresponds to the class $1 \mathrm{PBM}\left[\mathrm{X}(\mathrm{S} / \mathrm{T}) \mathrm{X}(\mathrm{L} / \mathrm{V} / \mathrm{I} / \mathrm{C})-\mathrm{COOH}\right.$, where $\mathrm{X}$ is any amino acid residue $\left.{ }^{34,35}\right]$. It has previously been shown that the E6 PBMs are susceptible to phosphorylation by protein kinases on the conserved Ser/Thr residue at the antepenultimate C-terminal position, which contributes to the class 1 PBM consensus ${ }^{18,21,23}$. This phosphosite is preceded by arginine residues in most of the HPV-E6 PBM sequences with recognizable patterns of the two common basophilic kinase substrate consensus motifs of $R(X / R) X(S / T)$ and $\mathrm{RXRXX(S/T)}{ }^{36,37}$. Additional Arg residues, e.g. at position -6 are most probably less involved in kinase recognition and more important for binding to other partners. For instance, Arg -6 of $18 \mathrm{E} 6$ is important for PDZ recognition but its mutation does not affect its phosphorylation efficiency by PKA, while mutation of Arg -5 and -4 blocks 18E6 PBM phosphorylation ${ }^{38}$. Considering the basic consensus motifs, these 31 E6 PBMs can be readily divided into three subgroups: Subgroups 1 and 2 having sequences prone to phosphorylation by the basophilic kinases and the third, orphan subgroup with a less predictable phosphorylation propensity (Fig. 1C). Accordingly, the PBM of HPV66 E6, which in our classification falls into the orphan group, is normally recalcitrant to phosphorylation by PKA in cellulo but becomes prone to it upon introduction of Arg residues at its positions -5 and $-4{ }^{38}$. In addition, more than half of the identified HPV-E6 PBM sequences harbor candidate phosphorylation sites of DNA damage response ATM/ATR kinases with a consensus of $(S / T) Q{ }^{39}$.

Therefore, the regulation of subgroup 1 and 2 E6 PBMs by phosphorylation is likely a rather general phenomenon. In line with earlier observations ${ }^{21,24,40}$, the phospho-PBM sequences from subgroups 1 and 2 can ideally match the C-terminal 14-3-3-binding motif III $^{30}$ (Fig. 1B and C). It is therefore likely that phosphorylation of the C-terminal PBMs from HPV-E6 types belonging to subgroups 1 and 2 by different kinases triggers their recognition by $14-3-3$ proteins ${ }^{18,21,22,24}$. 
156

157

158

159

160

161

162

163

164

165

166

167

168

169

170

171

172

173

174

175

176

177

178

179

180

181

182

183

184

185

186
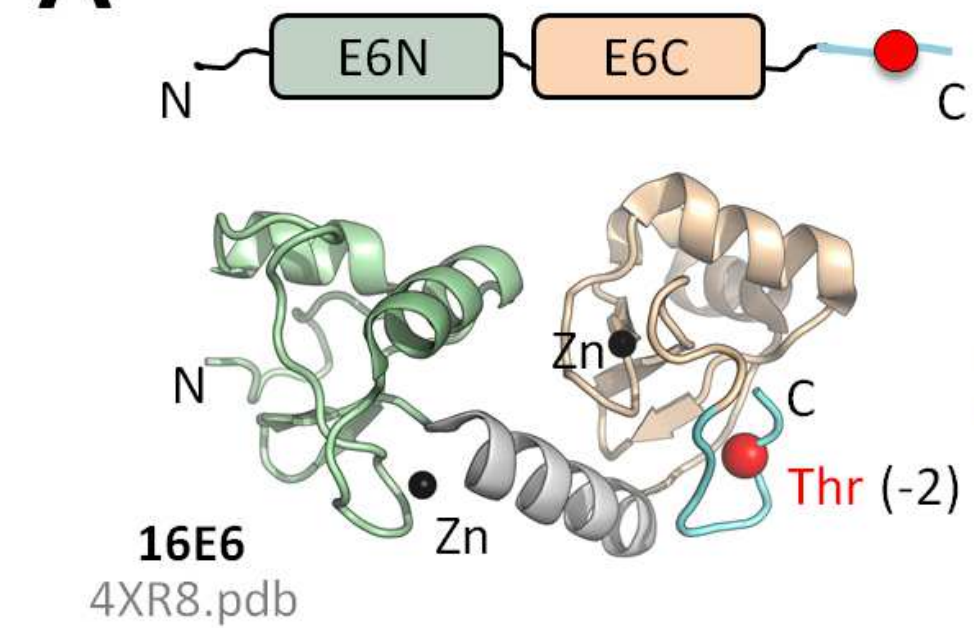

$-9-8-7-6-5-4-3-2-10$

...SSRTRRETQL-COOH $16 \mathrm{E} 6$ ...RLQRRRETQV-COOH $18 \mathrm{E} 6$ ...WRSRRRETAL- $\mathrm{COOH} \quad 33 \mathrm{E} 6$ ...WKPTRRETEV-COOH $35 \mathrm{E} 6$
14-3-3 binding motif III

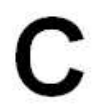

Subgroup 2

RXRXX $\underset{T}{\mathrm{~T}}$

$16 \mathrm{E} 6$

$70 \mathrm{E} 6$

$45 \mathrm{E} 6$

$31 \mathrm{E} 6$

$51 \mathrm{E} 6$

$52 \mathrm{E} 6$

$82 \mathrm{E} 6$

$68 \mathrm{E} 6$
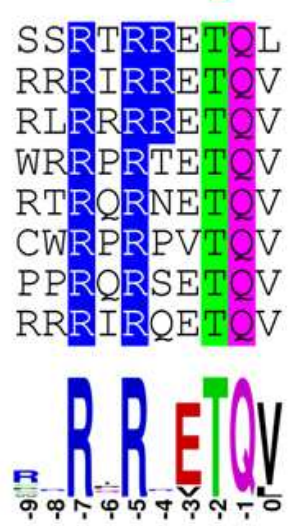

Orphan subgroup

$177 \mathrm{E} 6$ $26 \mathrm{E} 6$

$69 \mathrm{E} 6$

$30 \mathrm{E} 6$

$34 \mathrm{E} 6$

$53 \mathrm{E} 6$

$66 \mathrm{E} 6$

$56 \mathrm{E} 6$

$73 \mathrm{E} 6$
WRPTSQETQV

RPRRQTETQV

PRREATETQV

RHTTSTETAV

QCWRPSATVV

RHTTATESAV

TSRQATESTV

TSREPRESTV

RCWRPSATVV
$18 \mathrm{E} 6$

$97 \mathrm{E} 6$

$39 \mathrm{E} 6$

$85 \mathrm{E} 6$

$58 \mathrm{E} 6$

$67 \mathrm{E} 6$

$35 \mathrm{E} 6$

33E 6

$43 \mathrm{E} 6$

$91 \mathrm{E} 6$

$7 \mathrm{E} 6$

$40 \mathrm{E} 6$

$59 \mathrm{E} 6$

$89 \mathrm{E} 6$

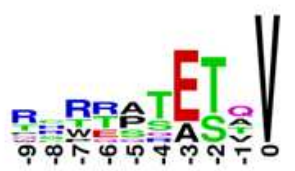

\section{Subgroup 1}

RXX
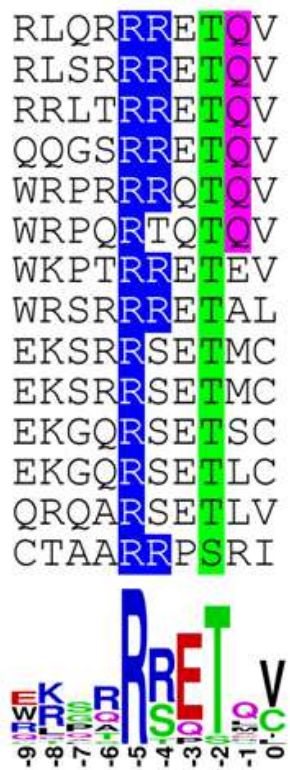

Fig. 1. Features of the structure and the C-terminal PDZ-binding motif of E6 proteins. A. The domain organization (top) and three dimensional structure (bottom) of HPV16 E6 (16E6) showing the location of the phosphorylatable PDZ-binding motif (PBM). N-terminal $(\mathrm{E} 6 \mathrm{~N})$ and $\mathrm{C}$-terminal $(\mathrm{E} 6 \mathrm{C})$ domains are color coded, structural $\mathrm{Zn}$ atoms are in black, PBM is in cyan, phosphorylatable Thr -2 is in red. B. E6 PBMs from high-risk mucosal HPV types overlap with the 14-3-3-binding motif $\mathrm{III}^{30}$. The positions are numbered above, according to conventional PBM numbering. C. Classification of 31 PBM-containing HPV-E6 proteins based on the correspondence of their C-terminal PBMs to the consensus motifs phosphorylatable by basophilic kinases (subgroups 1 and 2). The third, orphan subgroup comprises PBMs of HPV-E6 proteins whose phosphorylation is less certain. Bold font indicates the E6 PBMs used in this work. Note that many PBMs overlap with recognition motifs (S/TQ) for phosphorylation by DNA damage response kinases ATM/ATR (TQ and $\mathrm{SQ}$ sites highlighted). Below are shown Weblogo diagrams ${ }^{41}$ for the PBMs within each of the three subgroups (positions are numbered according to the PBM convention). 

seven 14-3-3 proteins with parallel binding profiles

190 Four phospho-PBMs from E6 proteins of HPV types 16, 18, 33 and 35 belonging to 191 subgroups 1 and 2 (as defined in Fig. 1C) were analyzed for their interaction with the all 192 seven human 14-3-3 homodimers. For comparison, we also measured two non-viral phospho-PBMs originating from protein kinase RSK1 ${ }^{24}$. We used a competitive fluorescence polarization assay that measures the displacement of a fluorescent tracer 143-3-binding peptide in the presence of an increasing amount of a competitor molecule (all

196 binding curves are shown in Supplementary Fig. 1A).

All phospho-PBMs (hereinafter called p16E6, p18E6, p33E6, p35E6, RSK1_-1P, and 198 RSK1_-2P) detectably bound to 14-3-3 proteins, in sharp contrast to their 199 unphosphorylated counterparts. The interactions between E6 phospho-PBMs and 14-3-3 200 proteins spanned very wide affinity ranges, from just below $1 \mu \mathrm{M}$ (p33E6-14-3-3y) to 201 above $300 \mu \mathrm{M}$ (Fig. 2A and Supplementary Fig. 1). Such large binding affinity differences are noteworthy since the four E6 PBM sequences are very similar (Fig. 1B), and all 14-3-3 isoforms share highly conserved phosphopeptide-binding grooves.

Remarkably, the six phospho-PBMs showed consistent hierarchy in their relative binding preferences towards any of the seven 14-3-3 isoforms, albeit with a systematic overall shift in affinity from one peptide to another. The seven 14-3-3 isoforms clustered as four groups of decreasing affinity, in a conserved order from the strongest to the weakest phosphoPBM binder: gamma, eta, zeta/tau/beta and epsilon/sigma ( $\gamma, \eta, \zeta / \mathrm{T} / \beta$, and $\varepsilon / \sigma)$ (Fig. 2B). These conserved relative affinity shifts can be quantified by calculating, for two distinct 143-3 isoforms, their differences of free energy of binding $(\Delta \Delta G)$ towards each individual phosphopeptide, then calculating the average difference $\left(\Delta \Delta G_{a v}\right)$ with its standard deviation (Fig. 2C). Between the strongest and the weakest binders (isoforms $\mathrm{Y}$ and $\sigma$, respectively) the average phosphopeptide-binding energy difference is $\Delta \Delta \mathrm{G}_{\mathrm{av}}=-5.1 \pm 1.3$

$214 \mathrm{~kJ} / \mathrm{mol}$, roughly corresponding to a 11 -fold $\mathrm{K}_{\mathrm{D}}$ ratio.

Noteworthy enough, another study ${ }^{42}$ also measured the binding of seven human 14-3-3 isoforms to the completely unrelated cystic fibrosis transmembrane conductance regulator (CFTR) phosphopeptide CFTR-R7, representing an internal 14-3-3-binding motif. A roughly comparable hierarchy of affinities was observed, also showing a 11-fold $K_{D}$ ratio between the strongest binder (gamma isoform, $\mathrm{K}_{D}=1 \mu \mathrm{M}$ ) and the weakest binder (epsilon isoform, $\left.\mathrm{K}_{\mathrm{D}}=11 \mu \mathrm{M}\right)$; both values being remarkably close to those obtained herein for the strongest-binding E6 phospho-PBM, p33E6. Similar trends were also detected upon interaction of the Leucine-Rich Repeat Kinase 2 (LRRK2) internal motif phosphopeptides with 14-3-3 isoforms, when gamma and eta were the strongest and epsilon and sigma were the weakest binders ${ }^{43}$. The $K_{D}$ ratio for the gamma and sigma isoforms averaged for the three of LRRK2 phosphopeptides $(\sim 20){ }^{43}$ is remarkably similar to the 11-fold difference described here for the E6 PBM interaction with the strongest and weakest human 14-3-3 isoforms. Therefore, our data support the idea that specificity of 14-3-3 isoforms for protein 228 partners follows a general trend.

229 The seven 14-3-3 isoforms also showed consistent profiles in their relative binding 230 preferences towards any of the four E6 phospho-PBMs. For each 14-3-3 isoform, the four 231 phospho-PBMs systematically rank the same way from the strongest to the weakest 232 binder: p33E6, p18E6, p16E6 and p35E6 (Fig. 2C). The average 14-3-3 binding free 
233 energy difference between p33E6 and p35E6 was $\Delta \Delta \mathrm{G}_{\mathrm{av}}=-10.9 \pm 0.7 \mathrm{~kJ} / \mathrm{mol}$, roughly

234 corresponding to a 100 -fold $K_{D}$ ratio.

A

\begin{tabular}{|c|c|c|c|c|c|c|c|}
\hline & \multicolumn{7}{|c|}{$K_{D}(\mu M) \pm s t d$} \\
\hline & $14-3-3 \gamma$ & $14-3-3 \eta$ & $14-3-3 \zeta$ & $14-3-3 \tau$ & $14-3-3 \beta$ & $14-3-3 \varepsilon$ & $14-3-3 \sigma$ \\
\hline pHPV33E6 & $0.94 \pm 0.25$ & $1.98 \pm 0.15$ & $2.72 \pm 0.39$ & $3.52 \pm 0.28$ & $4.05 \pm 0.65$ & $10.3 \pm 2.39$ & $6.77 \pm 1.13$ \\
\hline pHPV18E6 & $11.1 \pm 0.6$ & $23.6 \pm 6.2$ & $22.4 \pm 1.3$ & $37.8 \pm 6.0$ & $42.2 \pm 6.3$ & $101 \pm 20$ & $139 \pm 24$ \\
\hline pHPV16E6 & $37.2 \pm 3.0$ & $80.5 \pm 16.9$ & $73.6 \pm 19.1$ & $144 \pm 28$ & $159 \pm 45$ & $>300$ & $>300$ \\
\hline pHPV35E6 & $125 \pm 6$ & $163 \pm 19$ & $191 \pm 18$ & $233 \pm 14$ & $>300$ & $>300$ & $>300$ \\
\hline pHPV35E6 $_{T-6 R}$ & $71.2 \pm 6.7$ & $85.0 \pm 20.6$ & $139 \pm 21$ & $194 \pm 37$ & $179 \pm 29$ & $270 \pm 20$ & $>300$ \\
\hline pHPV35E6 $_{\text {E-1A }}$ & 0.72 & 12. & 3.6 & 21. & 1.4 & 4.2 & $44.1 \pm 13.3$ \\
\hline 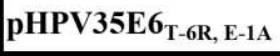 & $3.27 \pm 0.16$ & $4.77 \pm 0.53$ & $7.32 \pm 0.42$ & $5.84 \pm 0.43$ & $7.06 \pm 0.51$ & $13.0 \pm 2.0$ & $18.9 \pm 1.9$ \\
\hline RSK1_-1P & $0.31 \pm 0.03$ & $0.46 \pm 0.06$ & $0.96 \pm 0.10$ & $0.78 \pm 0.13$ & $0.74 \pm 0.04$ & $1.58 \pm 0.21$ & $1.71 \pm 0.23$ \\
\hline RSK1_-2P & $0.20 \pm 0.01$ & $0.66 \pm 0.05$ & $1.54 \pm 0.09$ & $0.45 \pm 0.08$ & $0.96 \pm 0.03$ & $1.22 \pm 0.16$ & $3.57 \pm 0.32$ \\
\hline
\end{tabular}

pHPV33E6

\section{\begin{tabular}{|l|l|l|l|l|l|l|}
$14-3-3 \gamma$ & $14-3-3 \eta$ & $14-3-3 \zeta$ & $14-3-3 \tau$ & $14-3-3 \beta$ & $14-3-3 \varepsilon$ & $14-3-3 \sigma$ \\
\hline
\end{tabular}}

\section{C $\quad \Delta \Delta \mathrm{G}_{\mathrm{av}}=-0.71 \pm 1.19 \quad-1.50 \pm 0.63 \quad-0.34 \pm 0.66 \quad-0.14 \pm 1.39 \quad-0.87 \pm 0.78 \quad-1.35 \pm 0.82(\mathrm{~kJ} / \mathrm{mol})$ $14-3-3 \sigma<14-3-3 \varepsilon<14-3-3 \beta<14-3-3 \tau<14-3-3 \zeta<14-3-3 \eta<14-3-3 \gamma$}

$$
\Delta \Delta \mathrm{G}_{\mathrm{av}}=\quad-2.06 \pm 0.67 \quad-3.09 \pm 0.15 \quad-5.98 \pm 0.65 \quad(\mathrm{~kJ} / \mathrm{mol})
$$

HPV35E6 < HPV16E6 < HPV18E6 < HPV33E6

Fig. 2. Summary of affinity measurements carried out in this study. A. Affinities of four selected HPV-E6 phospho-PBMs, p35E6 mutants and RSK1 phosphopeptides towards the seven human 14-3-3 isoforms as determined by fluorescence polarization using FITClabeled HSPB6 phosphopeptide as a tracer. Apparent $K_{D}$ values determined from competitive FP experiments are presented. B. The heatmap representation of the data on panel A showing the affinity trends in the interaction profiles between 14-3-3 isoforms and four HPV-E6 phospho-PBMs from weakest (white) to strongest (red). C. Averaged $\Delta \Delta G$ values between 14-3-3 isoforms or E6 phospho-PBM pairs, calculated based on their observed order of binding affinities (from weakest to strongest). Individual $K_{D}$ values from Supplementary Fig. 1 were first converted into $\Delta G$ values (at $T=295 \mathrm{~K}$; excluding cases when $\left.K_{D}>300 \mu \mathrm{M}\right)$ and average $\Delta \Delta G$ values $\left(\Delta \Delta G_{a v}\right)$ were calculated between the indicated motifs/isoforms.

\section{Atomic structure reveals the 14-3-3ろ / 18E6 PBM interface}

To get structural insight into the 14-3-3 3 interactions with 18E6 PBM, we designed, as previously reported ${ }^{44,45}$, a protein-peptide chimera, which we co-expressed with PKA and purified from E. coli cells by using an engineered cleavable His 6 tag (Supplementary Fig. $2 \mathrm{~A}-\mathrm{C})$. The 14-3-3 3 core was modified to block phosphorylation of the semiconserved Ser58 located in the dimer interface ${ }^{46}$ and to facilitate crystallization ${ }^{47}$ and was tethered to 
269 the 18E6 phosphorylatable peptide RRRETQV-COOH. This approach was justified 270 because of the disordered nature of the E6 PBMs ${ }^{48,49}$.

271 The 14-3-3ろ-18E6 PBM chimera was stoichiometrically phosphorylated by co-expressed 272 PKA, which gave a distinct downward shift on native PAGE (Supplementary Fig. 2D), and determination was carried out at a $1.9 \AA$ resolution with a single 14-3-3 dimer in the asymmetric unit (Supplementary Fig. 3A). The 18E6 phosphopeptide of this dimer was bound in-trans in the amphipathic 14-3-3 grooves of the crystallographic symmetry neighbor molecule, resulting in swapped phosphopeptides (Supplementary Fig. 3A). Such arrangement, previously observed for 14-3-30 chimeras with other phosphopeptides ${ }^{44,50}$ provided us with high-resolution insight into the 14-3-3/18E6 PBM interaction (Fig. 3A-C). All residues of the $18 \mathrm{E} 6$ phosphopeptides could be traced including the conformation of the C-terminal carboxyl-group. The phosphopeptide is stabilized by multiple polar interactions involving those between phospho-Thr156 (position -2) and conserved residues of the basic pocket in the amphipathic groove of 14-3-3 (Arg56, Arg129, Lys49 and Tyr128, 14-3-3ろ numbering), the phosphopeptide backbone interactions to Asn224 and Asn173, and a remarkable polar contact between the carboxyl-group of the peptide and the side chains of Lys 120 and one of the two alternative conformations of Ser45 of 14-3-3ろ (Fig. 3C). The carboxyl-group orientation is further stabilized by in-cis $\mathrm{H}$-bond with the side chain of the preceding residue Gln157 (position -1) (Fig. 3C). The side chain of the C-terminal Val158 (position 0 ) of the peptide makes a hydrophobic contact with Val46 of 14-3-3ろ. A second remarkable hydrophobic contact is observed between the side chain of Val176 of 14-3-3ろ and the methyl-group of phospho-Thr156 (position -2), which hints at a more stable binding of $\mathrm{pThr}$ than would be in the case of pSer.

Importantly, the conformation of $18 \mathrm{E} 6$ phosphopeptide bound to $14-3-3 \zeta$ within the chimera is practically identical (RMSD $=0.17 \AA$ upon superimposition of the six core Ca atoms of the peptides) to the 14-3-3б-bound conformation of a synthetic 16E6 phosphopeptide reported very recently at a much lower resolution (Fig. 3D) ${ }^{24}$. In both cases, the carboxylgroup gives a polar contact to the Lys120 side chain and the hydrophobic side chain of the last peptide residue (Val158 in 18E6 and Leu151 in 16E6) faces the side chain of Val46 of 14-3-3. This validates the chimeric approach ${ }^{44}$ as a strategy to obtain high-resolution crystal structures of 14-3-3/phosphopeptide complexes. Arg residues that are likely to be involved in kinase recognition also contribute to 14-3-3 binding. The Arg residue occupying position -4 forms an in-cis interaction with the phosphoryl group, and the Arg residue occupying position -5 forms in both 16E6 and 18E6 a m-stacking interaction with Arg60 of 14-3-3 (Fig. 3D). Most of interface contacts of the 18E6/14-3-3 3 and 16E6/14-3-30 complexes are similar, suggesting that the two current crystal structures can also serve as templates to build accurate homology models of other 14-3-3/E6 complexes and, more generally, any motif III complexes with a pS/pTXX-COOH consensus.

However, some noteworthy differences appear in a subset of the crystallographic conformers of 14-3-3/16E6 and 14-3-3/18E6 complexes. On the one hand, in 1 of the 4

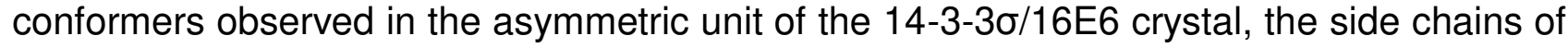
Arg -7 (Gln in 18E6) and Glu -3 form an additional in-cis salt bridge (Fig. 3D). On the other hand, Arg -6 of 18E6 (Thr in 16E6) mediates a bipartite interaction with 14-3-3 in most of the observed conformers. It simultaneously interacts with the carbonyl of Asp223 and participates in a water-mediated interaction with Asn224 (Fig. 3C and D). 

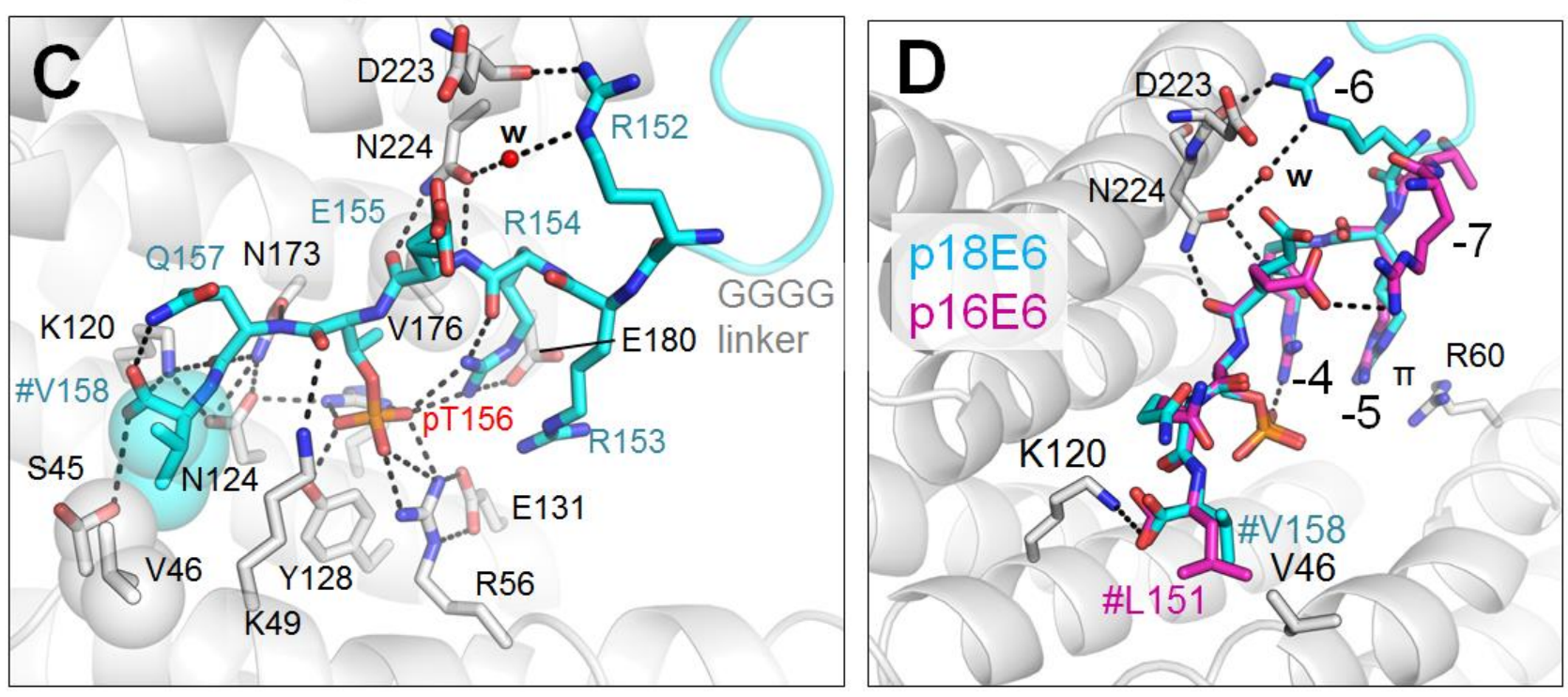

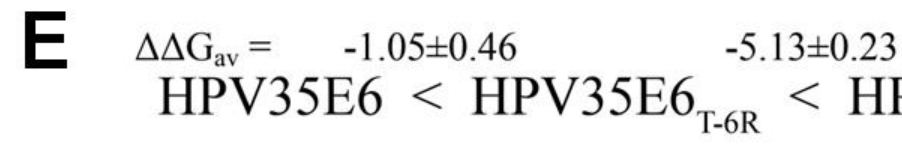

Fig. 3. Molecular interface between $14-3-3 \zeta$ and phosphorylated $18 \mathrm{E} 6 \mathrm{PBM}$ at a $1.9 \AA$ resolution. A. An overall view on the 14-3-3 3 dimer (subunits are in tints of grey) with two bound 18E6 phosphopeptides (cyan sticks). B. A magnified view on one of the amphipathic grooves of $14-3-3 \zeta$ showing the conformation of the 18E6 phosphopeptide and the corresponding $2 \mathrm{~F}_{\mathrm{o}}-\mathrm{F}_{\mathrm{c}}$ electron density maps contoured at $1 \sigma$. Positions are numbered according to the PBM convention. C. Polar contacts (dashed lines) and hydrophobic interactions (semitransparent spheres) stabilizing the bound 18E6 peptide conformation. $\mathbf{D}$. An overlay of the two 14-3-3 bound phosphopeptides from 16E6 (6TWZ.pdb) and 18E6 (this work) showing the similarity of the conformation. \# denotes the C-terminus $(-\mathrm{COOH})$. $\mathrm{w}-$ the water molecule, $\pi-\pi$-stacking interaction. Important positions are numbered according to the PBM convention. E. Averaged $\Delta \Delta G$ values between 14-3-3 isoforms or 35E6 phospho-PBM pairs, calculated based on their observed order of binding affinities (from weakest to strongest). Individual $K_{D}$ values from Supplementary Fig. 1 were first converted into $\Delta G$ values (at $T=295 \mathrm{~K}$; excluding cases when $K_{D}>300 \mu M$ ) and average $\Delta \Delta \mathrm{G}$ values $\left(\Delta \Delta \mathrm{G}_{\mathrm{av}}\right)$ were calculated between the indicated motifs/isoforms. 
350 We hypothesized that the remarkable affinity differences of the four studied E6 phospho351 PBMs $\left(\Delta \Delta \mathrm{G}_{\mathrm{av}}=-10.9 \pm 0.7 \mathrm{~kJ} / \mathrm{mol}\right.$ between the weakest and the strongest 14-3-3-binder $)$ 352 might result from two opposed mechanisms: interface peptide-14-3-3 contacts favoring complex formation versus intra-peptide contacts within the unbound peptide disfavoring it. On the one hand, position -6 is an Arg in the two strongest 14-3-3-binders (18E6 and 33E6) versus a Thr in the weakest ones (35E6 and 16E6). Accordingly, the crystal structures have shown that Arg -6 can mediate more interactions with the generic 14-3-3 interface (Fig. 3D). On the other hand, all E6 phospho-PBMs have a delicate charge distribution, with an acidic C-terminus (that is also involved in PDZ-domain binding) and a basic $\mathrm{N}$-terminal segment (that is also involved in kinase recognition). These local charged segments may form transient in-cis interactions within the unbound phosphopeptide, socalled "charge clamps" ${ }^{51}$, potentially unfavorable for 14-3-3 binding. We speculated that Glu -1 in p35E6, the weakest 14-3-3 binder, might participate in such a charge clamp, thereby disfavoring the binding.

364 To test these mechanisms, we synthesized three variants of p35E6. The first variant contained a T-6R substitution, which in principle could allow a more stable bound conformation, but may also stabilize charge-clamps in the free form of the motif. The second variant contained an E-1A substitution, which in principle could destabilize in-cis charge-clamps. A third variant contained both substitutions. All substitutions turned out to reinforce the binding affinities of 35E6 without altering the apparent preferences of the different 14-3-3 isoforms (Fig. 2A and Fig. 3E). Taken individually, T-6R moderately increased binding $\left(\Delta \Delta \mathrm{G}_{\mathrm{av}}=-1.1 \pm 0.5 \mathrm{~kJ} / \mathrm{mol}\right.$, 1.5-fold $\mathrm{K}_{\mathrm{D}}$ ratio), while $\mathrm{E}-1 \mathrm{~A}$ strongly reinforced it $\left(\Delta \Delta \mathrm{G}_{\mathrm{av}}=-5.1 \pm 0.2 \mathrm{~kJ} / \mathrm{mol}, 11\right.$-fold $\mathrm{K}_{\mathrm{D}}$ ratio). When combined, the two substitutions synergistically increased binding $\left(\Delta \Delta \mathrm{G}_{\mathrm{av}}=-8.7 \pm 0.4 \mathrm{~kJ} / \mathrm{mol}\right.$, 35-fold $\mathrm{K}_{\mathrm{D}}$ ratio), thereby turning p35E6 from the weakest 14-3-3 binder into the second strongest one, just below p33E6.

\section{Fusicoccin partially destabilizes the 14-3-3/E6 PBM interaction}

378 Fusicoccin (FSC) is commonly used as a stabilizer of 14-3-3 complexes, when its binding 379 in the distinct pocket in the 14-3-3/phosphopeptide interface is allowed by phosphopeptide 380 side chains of the amino acids in downstream positions relative to the phospho-residue ${ }^{42}$, $52,53,54$. Binding of FSC is known to stabilize the 14-3-3 interaction with few internal motif I phosphopeptides (e.g., Gab2 peptide, 5EXA.pdb ${ }^{55}$ and CFTR peptide, 5D3F.pdb ${ }^{42}$ ) and numerous peptides with the short motif III consensus, pS/pTX-COOH ${ }^{31,56,57}$. Most of the internal motif phosphopeptides, when bound to 14-3-3, apparently leave insufficient space for FSC and therefore hinder formation of the ternary complexes with FSC. Many motif III peptides with a consensus $\mathrm{pS} / \mathrm{pTX}-\mathrm{COOH}$ enable synergistic binding of the phosphopeptide and FSC, reinforcing the 14-3-3/phosphopeptide complex $31,52,53,57,58,59$. At the same time, very little is known about the effect of FSC on binding of the longer motif III peptides to 14-3-3, such as in the case of E6 PBMs.

392

We investigated whether binding of phosphorylated E6 PBM to 14-3-3 might be affected by this small molecule and analyzed its effect on the affinity of the E6 PBMs to 14-3-3. We used FP experiments to measure equilibrium binding affinity constants of complexes 
between the four HPV-E6 phosphopeptides and 14-3-3 isoforms $\zeta$ and $\gamma$, in the presence of $100 \mu \mathrm{M}$ FSC (Supplementary Fig. 1B and Fig. 4A). The addition of FSC consistently decreased by 1.5 to 2 fold the affinities of all interactions $\left(\Delta \Delta G_{a v}=-1.3 \pm 0.5\right.$ and $-1.8 \pm 0.4$ $\mathrm{kJ} / \mathrm{mol}$ for $\zeta$ and $\mathrm{\gamma}$, respectively) without altering the apparent preferences of the different peptides (Fig. 4A and B). Therefore, fusicoccin moderately destabilized all studied 14-33/E6 interactions, in contrast to its much broadly reported, stabilizing effect on 14-3-3 complexes ${ }^{31,42,52}$. Nevertheless, the binding of E6 peptides and FSC was not mutually exclusive.

\begin{tabular}{|l|c|c|c|c|}
\cline { 3 - 5 } \multicolumn{1}{|c|}{} & \multicolumn{4}{c|}{$\mathrm{K}_{\mathrm{D}}(\mu \mathrm{M}) \pm$ std } \\
\cline { 2 - 5 } \multicolumn{1}{c|}{} & $\mathbf{1 4 - 3 - 3 \zeta}$ & $\mathbf{1 4 - 3 - 3 \zeta}+$ FSC & $\mathbf{1 4 - 3 - 3} \boldsymbol{\gamma}$ & $\mathbf{1 4 - 3 - 3} \boldsymbol{\gamma}+$ FSC \\
\hline pHPV33E6 & $2.72 \pm 0.39$ & $3.94 \pm 0.40$ & $0.94 \pm 0.25$ & $2.15 \pm 0.26$ \\
\hline pHPV18E6 & $22.4 \pm 1.3$ & $37.3 \pm 5.2$ & $11.1 \pm 0.6$ & $19.3 \pm 2.0$ \\
\hline pHPV16E6 & $73.6 \pm 19.1$ & $161 \pm 23$ & $37.2 \pm 3.0$ & $91.7 \pm 13.2$ \\
\hline pHPV35E6 & $191 \pm 18$ & $294 \pm 15$ & $125 \pm 6$ & $228 \pm 61$ \\
\hline
\end{tabular}

$$
\begin{aligned}
& \Delta \Delta \mathrm{G}_{\mathrm{av}}=\quad-1.28 \pm 0.45(\mathrm{~kJ} / \mathrm{mol}) \\
& 14-3-3 \zeta+\mathrm{FSC}<14-3-3 \zeta \\
& \Delta \Delta \mathrm{G}_{\mathrm{av}}=\quad-1.77 \pm 0.42 \quad(\mathrm{~kJ} / \mathrm{mol}) \\
& 14-3-3 \gamma+\mathrm{FSC}<14-3-3 \gamma
\end{aligned}
$$
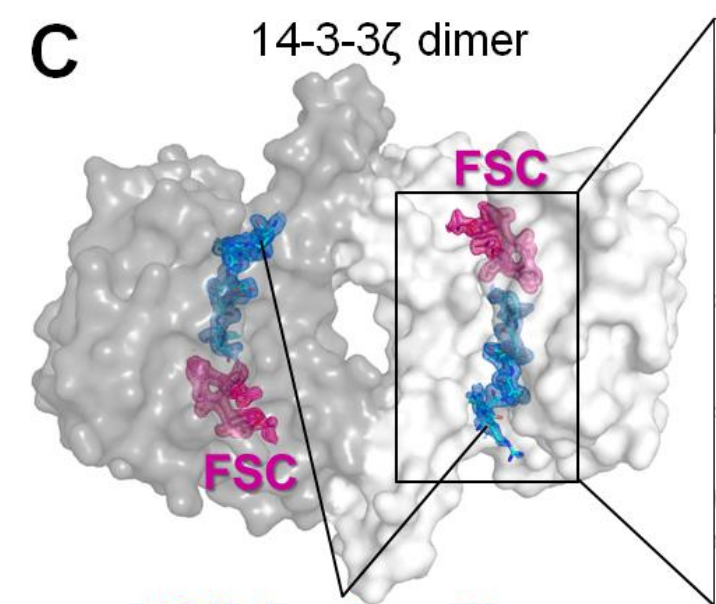

18E6 phosphopeptides
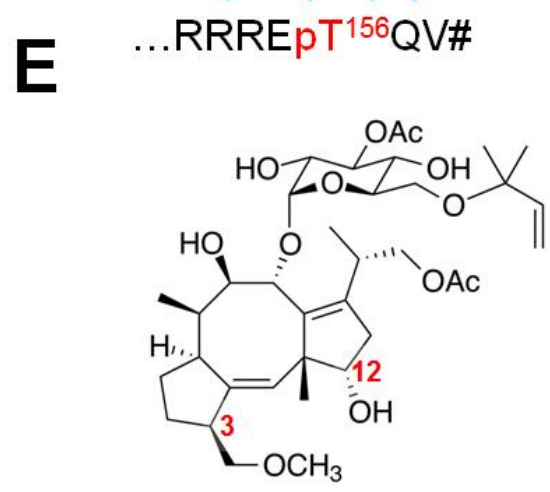

\section{Fusicoccin A (FSC)}

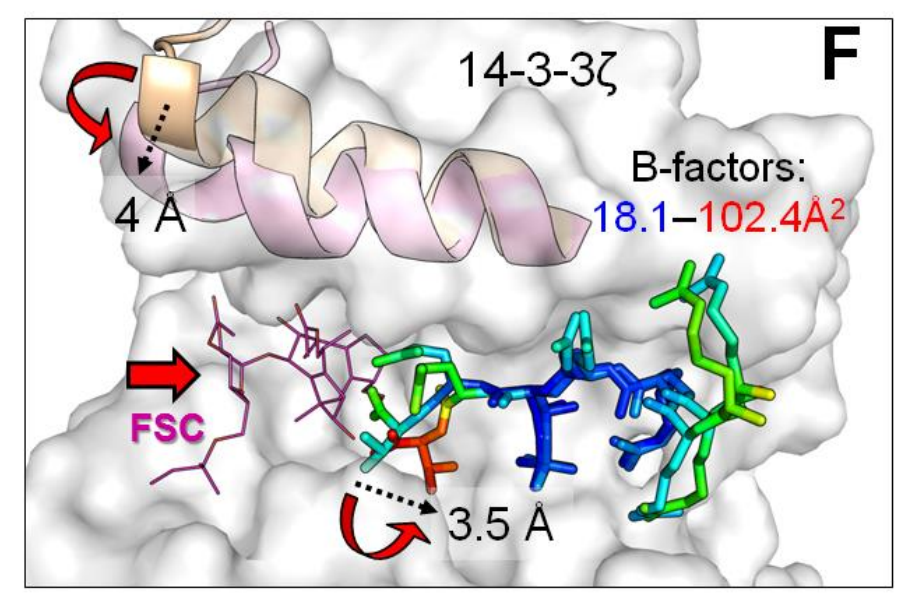

Fig. 4. The destabilizing effect of FSC on the 14-3-3Z/18E6 PBM interaction. A. Affinities of four selected HPV-E6 phospho-PBMs towards human 14-3-3 3 and 14-3-3y in the absence and in the presence of FSC as determined by fluorescence polarization using FITC-labeled HSPB6 phosphopeptide as a tracer. Apparent $K_{D}$ values determined from competitive FP experiments are presented. The binding curves are shown in Supplementary Fig. 1. B. Averaged $\Delta \Delta G$ values between 14-3-3-E6 phospho-PBM pairs in the absence or in the presence of FSC, calculated based on their observed order of binding affinities (from 
weakest to strongest). Individual $K_{D}$ values from Supplementary Fig. 1 were first converted into $\Delta \mathrm{G}$ values (at $\mathrm{T}=295 \mathrm{~K}$; excluding cases when $\mathrm{K}_{\mathrm{D}}>300 \mu \mathrm{M}$ ) and average $\Delta \Delta \mathrm{G}$ values $\left(\Delta \Delta \mathrm{G}_{\mathrm{av}}\right)$ were calculated between the indicated motifs/isoforms. C. An overall view on the ternary complex between 14-3-3ろ (subunits are shown by surface using two tints of grey), 18E6 phosphopeptide (cyan sticks) and FSC (pink sticks). FSC was soaked into the 14-3$3 \zeta-18 \mathrm{E} 6$ chimera crystals. $2 \mathrm{~F}_{\mathrm{o}}-\mathrm{F}_{\mathrm{c}}$ electron density maps contoured at $1 \sigma$ show are shown for the phosphopeptide and FSC only. D. A closeup view showing polar contacts (dashed lines) and hydrophobic interactions (semitransparent spheres) positioning the 18E6 phosphopeptide (cyan sticks) and FSC (thin pink sticks) in the amphipathic groove of the 14-3-3 $\zeta$ subunit (semitransparent light grey ribbon). $2 F_{0}-F_{c}$ electron density maps contoured at $1 \sigma$ are shown for the peptide and FSC. \# denotes the C-terminus $(-\mathrm{COOH})$. The GGGG linker is omitted for clarity. E. Chemical formula of FSC showing the positions of the functional groups discussed in the text. F. The effect of FSC binding. Conformational changes upon FSC binding are shown by red arrows reflecting the closure of the Cterminal $\alpha$-helix of 14-3-3 and the rotation and translocation of the C-terminal residues in the $18 \mathrm{E} 6$ peptide, accompanied by a significant rise of the local B-factors (are shown as a gradient from blue to red as indicated). The amplitudes of the conformational changes in 14-3-3 and 18E6 peptide are indicated in $\AA$ by dashed arrows.

\section{Structural basis for the destabilizing effect of fusicoccin}

To get structural insights into the peculiar destabilizing effect of FSC on the 14-3-3/E6 PBM interaction, we soaked the 14-3-3//18E6 PBM chimera crystals with FSC and solved the crystal structure of the 14-3-3ろ/18E6 PBM/FSC ternary complex (Fig. 4C-F and Table 1). FSC soaking did not disrupt the crystal lattice of the 14-3-3Z/18E6 PBM chimera crystals (Supplementary Fig. 3) and the overall assembly (Fig. 4C and D); however, significant local structural rearrangements could be observed. FSC (Fig. 4E) binding results in a $\sim 4 \AA$ closure of the last $\alpha$-helix of 14-3-3 $($ Fig. 4F), similar to what has already been described for other 14-3-3 complexes containing FSC ${ }^{58}$. Crystal lattice obtained by FSC soaking preserved the characteristic phosphopeptide swap stabilizing the contacts with the neighboring chimera molecules, and FSC was bound in each 14-3-3 $\zeta$ subunit, side-to-side with the C-terminal end of the 18E6 PBM (Fig. 4C and D, Supplementary Fig. 3). All residues and atoms within the analyzed area could be observed in the electron density thanks to the high resolution (Table 1).

460 FSC occupies its well-defined cavity where it is positioned by hydrophobic interactions with Phe117, Ile166, Ile217 and Leu216, polar contacts with residues Asn42 and Asp213, and a remarkable $\mathrm{H}$-bond involving its 3-metoxy oxygen and the side chain of Lys 120 of 14-3-3ろ (Fig. 4D). The latter contact breaks the Lys120 interaction with the carboxyl-group of the 18E6 PBM formed in the absence of FSC, displacing the carboxyl to another position, where it establishes a new contact with the 12-hydroxy group of FSC (Fig. 4D and E). 14-3$3 \zeta$ Lys49 also switches its position, and loses a contact to the backbone carbonyl of pThr156 (Fig. 3C) to establish instead a contact with the 12-hydroxy group of FSC. The side chain of the C-terminal Val158 of the 18E6 PBM shifts $3.5 \AA$ towards the phosphate moiety of Thr156, breaking the hydrophobic contact with Val46 of 14-3-3 $\zeta$ and significantly dispersing the local electron density (Fig. 4F). As a result, while most of the peptide conformation remained unchanged, the B-factors of the last 18E6 PBM residue in the refined FSC-bound structure increased significantly (Fig. 4F). Therefore, FSC binding 
causes an apparent strain in the conformation of the C-terminus of 18E6 PBM (Figs 4 and 5). In addition, the presence of FSC significantly reshuffles the water network that surrounds the E6 carboxy-terminal extremity, which directly contacts the bound FSC (Fig. 5). The simultaneous binding of the 18E6 PBM and FSC in the amphipathic groove of 14-33 and the mild destabilizing influence of FSC indicate that this ternary complex can be used as a starting point to design both stabilizers and inhibitors of 14-3-3/E6 interactions.
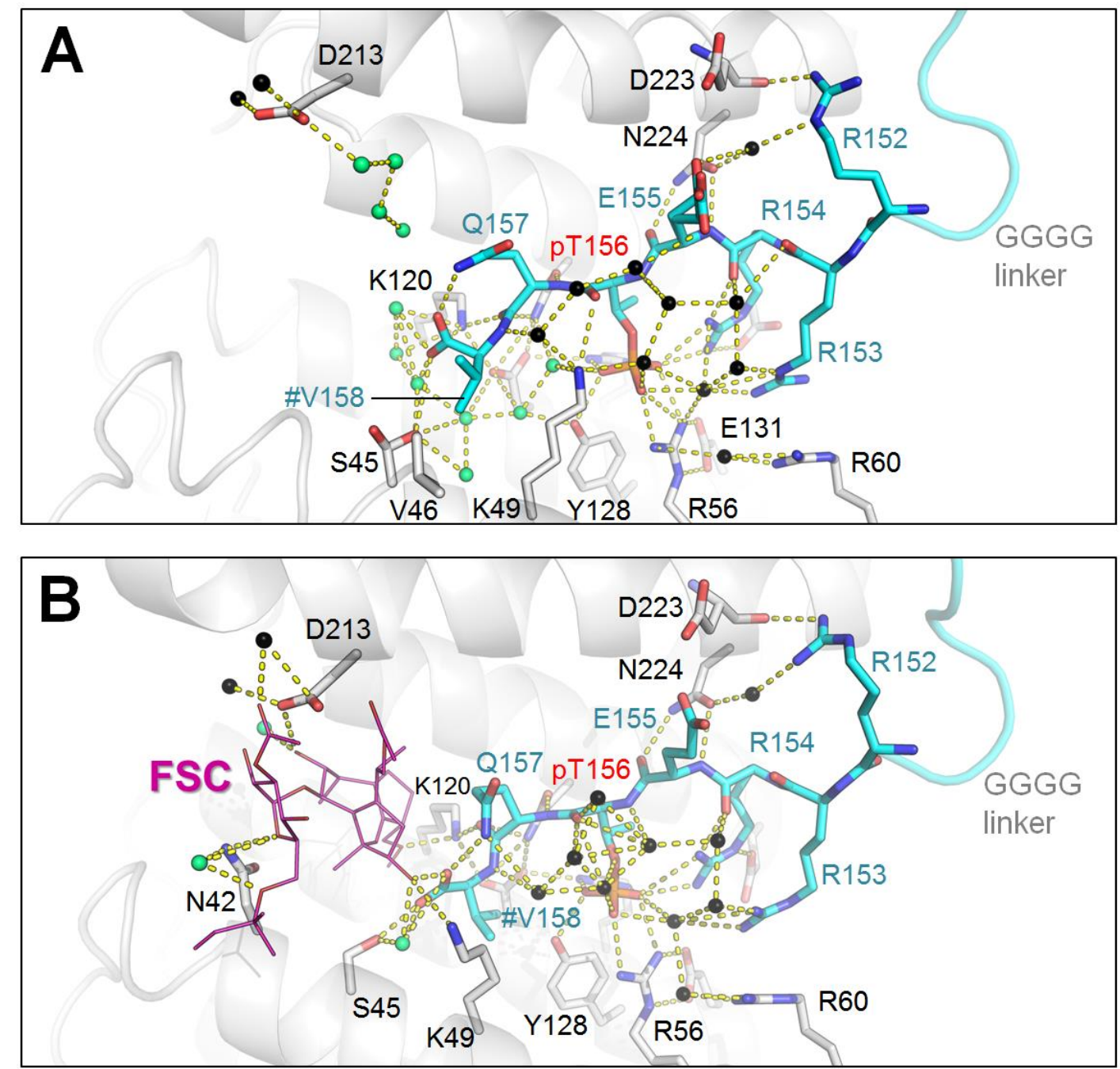

Fig. 5. Comparison of the water-mediated polar contacts formed in the 14-3-3ろ/18E6 interface in the absence (A) or in the presence of fusicoccin (FSC) (B). The main residues involved in the interactions are shown by sticks with color coding: 14-3-3 residues are shown in light grey, $18 \mathrm{E} 6$ residues are in cyan, phospho-group of Thr156 is shown by orange sticks. FSC is shown by thin magenta sticks, water molecules affected by FSC binding are shown by lime green, those similar in two structures are black. The C-terminal $18 \mathrm{E} 6$ residue (V158) is denoted by \#. Note the significant redistribution of water molecules upon FSC binding.

\section{DISCUSSION}

E6 oncoproteins of all high-risk alpha HPV types contain a conserved C-terminal PDZBinding Motif which can turn into a potential 14-3-3-binding motif upon phosphorylation of 
the conserved Thr/Ser residue at position -2 (Fig. 1). We demonstrated here that the phosphorylated forms of four selected alpha HPV-E6 PBMs detectably bound to all seven human 14-3-3 isoforms. Unexpectedly, the binding affinities of these 28 distinct 14-3-3/E6 complexes showed large variations, spanning a 10-fold $\mathrm{K}_{\mathrm{D}}$ range for different 14-3-3 isoforms binding to a given PBM, and a 100-fold $K_{D}$ range for different $E 6$ phospho-PBMs binding to a given 14-3-3 isoform. Furthermore, affinity variations followed trends that were all but random. The 14-3-3 binding profiles of all E6 phosphopeptides turned out to be remarkably parallel, and so were the E6-binding profiles of all 14-3-3 isoforms (Fig. 2B and C).

When considering the present data together with previously published literature, 14-3-3 interactions with phosphorylated motifs from different origins have a strikingly wide affinity range, spanning from low nanomolar to low millimolar detectable dissociation constants (Fig. 6). Despite variable interaction modes (e.g. monovalent vs. divalent), the 14-3-3 family-wide affinity trends are surprisingly parallel, with 14-3-3y and 14-3-3n consistently being the strongest binders and $14-3-3 \sigma$ and $14-3-3 \varepsilon$ being the weakest binders. Independently of the nature of the target motif, the average maximal $\mathrm{K}_{\mathrm{D}}$ ratio between the strongest-binding and the weakest-binding 14-3-3 is around 10-fold in our present work and also in the literature (Fig. 6), Conversely, phosphopeptides, even very similar in sequence, can sample much wider ranges of binding affinities for a given 14-3-3 isoform. For instance, for $14-3-3 \gamma$, the $K_{D}$ ratio between the strongest and the weakest binding phosphopeptide is almost 625-fold in the present work, and 39,000-fold when taking into account other reports $42,43,60,61$ (Fig. 6). However, the discussed weak interactions do not necessarily lead to the absence of complex formation in cellulo, as 14-3-3 proteins are among the most abundant proteins in human cells 62, 63. Indeed, according to Protein Abundance Database ${ }^{63}, 14-3-3 \varepsilon$ is the $48^{\text {th }}$ most abundant human protein (2479 ppm) and $14-3-3 \zeta$ is the $72^{\text {nd }}(1680 \mathrm{ppm})$, whereas all seven 14-3-3 isoforms are within the 820 most abundant proteins (i.e., the top $4.1 \%$ ) out of 19949 proteins in the integrated whole human body dataset. Given such high abundance, strong 14-3-3 interactions might lead to extremely tight complex formation, while weak interactions might lead to transient, more dynamic 14-3-3 complexes.

The observed strong and parallel affinity variations are noteworthy if one considers the high degree of conservation of the amphipathic grooves of the seven 14-3-3 isoforms and of the amphipathic groove-binding residues of the four E6 PBMs. Indeed, affinity differences may stem not only from structural particularities of the bound complexes but also from intrinsic properties of the unbound partners. Conformations unfavorable for complex formation may exist in various proportions for each 14-3-3 isoform and each E6 phosphopeptide, thereby influencing at various degrees the formation of the complexes and their resulting binding affinities. On the one hand, full-length 14-3-3 proteins feature the flexible C-terminal tails that are the most variable elements among isoforms; at least for some isoforms they have been shown to sample conformations also occupying the amphipathic 14-3-3 grooves and thereby regulating their affinities for target peptides ${ }^{64,65}$. Conformational dynamics of the 14-3-3 dimers along the open-closed state trajectory ${ }^{26,44}$ may also differ among 14-3-3 isoforms, which can probably affect the effectiveness of adopting the peptide-bound conformation. On the other hand, it has been shown that a phosphoryl-group in a disordered segment can form local "charge clamps" in-cis with a neighboring Arg/Lys residue, thereby decreasing its apparent availability for binding partners ${ }^{51}$. The four E6 PBMs studied herein display distinctive charged, polar or non-polar residues at several 
positions $(-1 ;-6 ;-7 ;-8 ;-9)$ that may influence their explorable conformational spaces. By replacing the Glu -1 by Ala in the weakest 14-3-3 binder, p35E6, we obtained a significantly stronger 14-3-3 binder, suggesting that we successfully disrupted a charge clamp that is unfavorable to binding of the p35E6 motif. Nonetheless, fine differences of contact networks at the interfaces of E6/14-3-3 complexes may also influence affinity. In this line, comparison of the present $\mathrm{p} 18 \mathrm{E} 6 / 14-3-3 \zeta$ structure to the previously solved $\mathrm{p} 16 \mathrm{E} 6 / 14-3-3 \sigma$ structure ${ }^{24}$ suggested that an Arg residue at position -6 established favorable interface contacts (Fig. 3C and D). Accordingly, replacing Thr -6 of p35E6 with Arg -6 as found in p18E6 and p33E6, effectively reinforced the binding event. Finally, combining both Glu $\rightarrow$ Ala and $\mathrm{Thr} \rightarrow$ Arg substitutions at positions -1 and -6 , respectively, resulted in a p35E6 variant that bound 14-3-3 proteins almost as strongly as the strongest studied E6 binder, p33E6 (Fig. 2A and Fig. 3E). Interestingly, the RSK1 phospho-PBMs (RRVRKLPSTpTL-COOH and RRVRKLPSpTTL-COOH), featuring non-charged residues Thr and Leu in the downstream position relative to the phosphothreonine, and therefore being unable to form the equivalent charge-clamp that is suggested in the case of p35E6 (SKPTRREpTEV-COOH), show remarkably high affinities to all 14-3-3 isoforms per se (Fig. 2A and Supplementary Fig. 1). Our data prove that charge clamps can indeed strongly contribute to the binding preferences of different phosphopeptide motifs. This principle needs to be further examined for all kinds of phosphorylation-regulated interactions as charge-clamp formation might be an often ignored biochemical property of practically all phosphorylation sites.

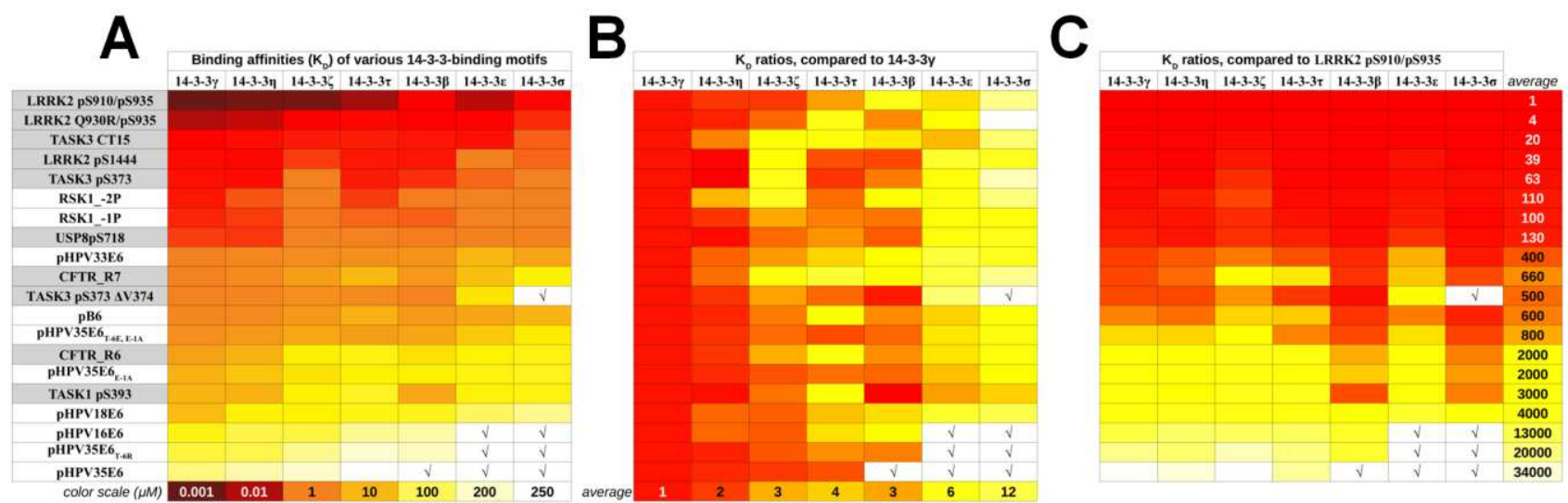

Fig. 6. Compilation of 14-3-3 family-wide affinity measurements of exemplary 14-3-3-binding motifs. A. Affinity maps of 14-3-3 interactions based on experimentally determined dissociation constants against the 14-3-3ome, as obtained in the current work and in refs $42,43,60,61$. B. When normalized to the strongest partner-binder 14-3-3y, all studied peptides are following very similar affinity trends between the different 14-3-3 isoforms. Between the strongest and the weakest partner-binding 143-3 isoform, a 12-fold dissociation constant decrease was found on average. C. When normalizing to the generally strongest studied 14-3-3-binding motif, the isoform-specific affinity variations for each peptide are compensated to clearly show the average differences between different binding motifs. Between the strongest and the weakest analyzed 14-3-3-binding motif, a 34,000-fold $K_{D}$ decrease was found on average for all 14-3-3 isoforms. Only counting the very similar HPV-E6 peptides sequence-wise, this affinity difference can still be larger than 100-fold. Therefore, the motif-to-motif affinity differences are expected to be much higher than the 14-3-3 isoform-to-isoform affinity differences. Binding motifs that are analyzed in other studies are highlighted with a grey background ${ }^{42,43,60,61}$. The color scale is either based on affinity values or in $K_{D}$ ratios. $\sqrt{ }$ denotes affinities weaker than the limit of quantitation of the fluorescence polarization assay. 
599 When phosphorylated, all the E6 PBMs studied here ideally match motif III consensus 600 recognized by 14-3-3 with two C-terminal residues downstream of the phosphorylated 601 threonine (pS/pTXX-COOH) (Fig. 1B). Structures of motif III complexes with 14-3-3 have 602 been less documented than the internal motif I/II complexes. Moreover, the pS/pTXX$603 \mathrm{COOH}$ subtype of motif III sites, studied here, is underrepresented in the Protein Data 604 Bank, where mostly motif III sites with only one residue after a phospho-residue have been 605 606 co-crystallized with 14-3-3 (Supplementary Table 1). To the best of our knowledge, the only two reported structures with $\mathrm{pS} / \mathrm{pTXX}-\mathrm{COOH}$ consensus peptides are those of 14-3-3y with the influenza virus protein NS1 (4O46.pdb) and our recently reported 14-3-3 $\sigma$ complex with the synthetic papillomavirus 16E6 phosphopeptide (6TWZ.pdb ${ }^{24}$ ), however, both of them have rather low resolution (2.8-2.9 $\AA$ ) (Supplementary Table 1). Together with the henipavirus protein $\mathrm{W}$ binding to $14-3-3 \sigma$ via its C-terminal motif III peptide RRMpSN$\mathrm{COOH}^{66}$, the adeno-associated virus protein Rep68 binding to 14-3-3 proteins using its Cterminal motif III peptide RGHpSL-COOH ${ }^{67}$ and the hepatitis $\mathrm{B}$ virus protein $\mathrm{X}$ binding to $14-3-3 \zeta$ via an internal motif I peptide RPLpSGP ${ }^{68}$, these examples illustrate that viral proteins commonly use their elements, efficiently mimicking the host 14-3-3-binding peptides, to hijack the cellular functions controlled by 14-3-3 proteins. One of the possible mechanisms is the 14-3-3-mediated stabilization of viral proteins to evade dephosphorylation and degradation, which may prolong the half-life and increase chances for successful replication and further infections ${ }^{21,68}$.

The phospho-PBM conformation revealed by our crystal structures, ideally congruous to the amphipathic groove of 14-3-3, leaves vacant the cavity that is known to be druggable and occupied by a fungal toxin called fusicoccin (FSC) ${ }^{33,54,56}$. Benefitting from the welldiffracting crystals of the 14-3-3弓-18E6 PBM chimera, we proceeded with their soaking with FSC, which resulted in a $1.85-\AA$ crystal structure of the ternary 14-3-3Z/18E6 PBM/FSC complex. The direct comparison of the 14-3-3//18E6 complex in the absence and in the presence of FSC revealed a significant conformational change in the last $\alpha$-helix of 14-3$3 \zeta$, a rearrangement of bound water molecules and a remarkable strain in the C-terminal part of the 18E6 PBM peptide (Figs 4 and 5). This strain forced the translocation of the carboxyl-group and increased the displacement factors for the last C-terminal residue, suggesting destabilization of the 14-3-3ろ/18E6 PBM complex by FSC. This partial destabilization, and thus the less-documented inhibitory action of FSC, was confirmed by in vitro binding assays using fluorescence polarization (Fig. 4A and B). Noteworthily, the structural data on the effect of FSC on the C-terminal end of the E6 PBM is very consistent with the preservation of the affinity differences for various 14-3-3/phospho-PBMs complexes in the presence of FSC (Fig. 4A). This supports the notion that those affinity differences are dictated by positions beyond the last $\mathrm{C}$-terminal residue, including those involved in formation of charge clamps.

We could find only one earlier reported example when FSC decreased the binding affinity of a 14-3-3 to a motif III peptide (the interleukin 9 receptor alpha chain (IL-R9a) peptide, RSWpTF- $\mathrm{COOH}{ }^{31}$ ), but in that case no experimental structural information was available. In addition, it was reported that binding of the shortest motif III peptide from the cyclindependent kinase inhibitor (p27 ${ }^{\mathrm{Kip} 1}$ ) RRQpT-COOH to 14-3-3 is not affected by FSC, most likely due to the absence of direct contacts between FSC and the phosphopeptide, which both bind in the amphipathic groove of 14-3-3 independently ${ }^{31}$. The structural basis for the inhibitory action of FSC has very recently been reported for several internal motif I 14-3-3- 
645 binding peptides ${ }^{58}$. Therefore, complementing the range of reports on the stabilizing effect 646 of FSC $31,42,54,55,56$, our results provide the first structural evidence that FSC can be a 647 negative regulator of 14-3-3 interactions with typical motif III peptides.

648 Thus, our structural and in vitro binding data with FSC confirm the druggability of the 14-3649 3-E6 interaction and suggest that appropriate modification and optimization of the small 650 molecule may provide promising opportunities for selective modulation of viral protein-14-36513 interactions in the future.

\section{METHODS}

653 Cloning, protein expression and purification and peptide synthesis

654 Previously described chimeras contained the C-terminally truncated human 14-3-30

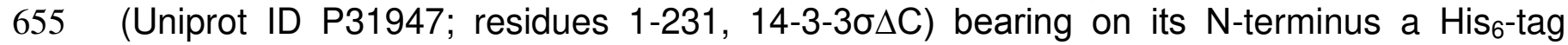
656 cleavable by $3 \mathrm{C}$ protease and phosphorylatable peptides tethered to the $14-3-3 \sigma \mathrm{C}$ 657 terminus by a GSGS linker ${ }^{44}$. The novel chimera was designed taking into account the 658 following modifications. First, it contained the C-terminally truncated human 14-3-3 $\zeta$ 659 sequence (Uniprot ID P63104; residues 1-229, 14-3-3Z $\Delta$ C) connected to the PKA-

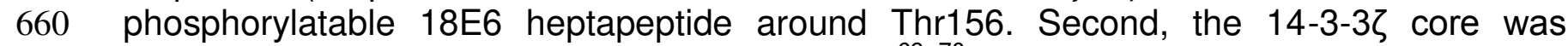
661 modified to block Ser58 phosphorylation (S58A) ${ }^{69,70}$. Third, to improve crystallizability, the $66214-3-3 \zeta$ sequence was mutated by introducing the ${ }^{73} \mathrm{EKK}^{75} \rightarrow \mathrm{AAA}$ and ${ }^{157} \mathrm{KKE}^{159} \rightarrow \mathrm{AAA}$ 663 amino acid replacements in the highest-scoring clusters 1 and 2 predicted by the surface 664 entropy reduction approach ${ }^{47,71}$. Finally, the linker was changed to GGGG to exclude its 665 unspecific phosphorylation (Supplementary Fig. 2A).

666 cDNA of the 14-3-3ל-18E6 chimera was codon-optimized for expression in Escherichia coli 667 and synthesized by IDT Technologies (Coralville, lowa, USA). The 14-3-3ろ $\Delta$ C gene was 668 flanked by Ndel and Agel restriction endonuclease sites to enable alteration of the 14-3-3 669 or E6 PBM peptide sequences. The entire 14-3-37-GGGG-18E6 PBM construct was 670 inserted into a pET28-his-3C vector ${ }^{72}$ using $\mathrm{Ndel}$ and $\mathrm{Xhol}$ restriction endonuclease sites. 671 The resulting vector was amplified in DH5a cells and verified using DNA sequencing in 672 Evrogen (Moscow, Russia, www.evrogen.ru).

673 The assembled vector (Kanamycin resistance) was transformed into chemically competent 674 E. coli BL21(DE3) cells for expression either in the absence or in the presence of the His ${ }_{6-}$ tagged catalytically active subunit of mouse PKA ${ }^{72}$. Protein expression was induced by the addition of isopropyl- $\beta$-thiogalactoside (IPTG) to a final concentration of $0.5 \mathrm{mM}$ and continued for $16 \mathrm{~h}$ at $25{ }^{\circ} \mathrm{C}$. The overexpressed protein was purified using subtractive immobilized metal-affinity chromatography (IMAC) and gel-filtration essentially as described earlier for $14-3-3 \sigma$ chimeras ${ }^{44}$ (Supplementary Fig. $2 \mathrm{~B}$ and $\mathrm{C}$ ). The purified phosphorylated 14-3-3弓-18E6 chimera revealed the characteristic downward shift on native PAGE compared to the unphosphorylated counterpart (Supplementary Fig. 2D). Given the absence of PKA phosphorylation sites in the modified 14-3-3Z core and the linker, this strongly indicated 18E6 phosphorylation by co-expressed PKA. The chimera was fully soluble and stable at concentrations above $20 \mathrm{mg} / \mathrm{ml}$ required for crystallization. Protein concentration was determined at $280 \mathrm{~nm}$ on a Nanophotometer NP80 (Implen, Germany) using extinction coefficient equal to $0.93\left(\mathrm{mg} / \mathrm{ml}^{-1} \mathrm{~cm}^{-1}\right.$.

For affinity measurements, full-length human 14-3-3 constructs with a rigid N-terminal MBP fusion were used. The coding sequences of the full-length 14-3-3 epsilon, gamma and zeta 
689

690

691

692

693

694

695

696

697

698

699

700

701

702

703

704

705

706

707

708

709

710

711

712

713

714

715

716

717

718

719

720

721

722

723

724

725

726

727

728

729

730

731

732

were received from Prof. Lawrence Banks. cDNAs encoding other full-length 14-3-3 isoforms $\beta, T, \eta$ and $\sigma$ were obtained as codon-optimized for E.coli expression synthetic genes from IDT Technologies (Coralville, lowa, USA). All 14-3-3 isoforms were fused via a three-alanine linker to the C-terminus of a mutant MBP carrying the following amino acid substitutions: D83A, K84A, K240A, E360A, K363A and D364A, as previously described ${ }^{49}$. All resulting clones were verified by sequencing. The MBP-fused proteins were expressed in E.coli BL21 with IPTG induction. Proteins were affinity purified on an amylose column and were further purified by ion-exchange chromatography (HiTrap Q HP, GE Healthcare). Protein concentrations were determined by UV spectroscopy. The double-purified samples were supplemented with glycerol and TCEP before aliquoting and freezing in liquid nitrogen.

HPV peptides (35E6: biotin-ttds-SKPTRRETEV; 16E6: biotin-ttds-SSRTRRETQL; 18E6: biotin-ttds-RLQRRRETQV; 33E6: biotin-ttds-SRSRRRETAL; p35E6: biotin-ttdsSKPTRREpTEV; p35E6 E-1A: biotin-ttds-SKPTRREpTAV; p35E6 T-6R: biotin-ttdsSKPRRREpTEV; p35E6 E-1A T-6R: biotin-ttds-SKPRRREpTAV; p16E6: biotin-ttdsSSRTRREpTQL; p18E6: biotin-ttds-RLQRRREpTQV; p33E6: biotin-ttds-SRSRRREpTAL) and RSK1 peptides (RSK1_-1P: biotin-ttds-RRVRKLPSTpTL and RSK1_-2P: biotin-ttdsRRVRKLPSPTTL) were chemically synthesized in-house on an ABI 443A synthesizer with Fmoc strategy. The fluorescently labeled HSPB6 (WLRRApSAPLPGLK) peptide (fpB6) was prepared by FITC labeling of the chemically synthesized peptide as described previously ${ }^{24}$.

\section{Fluorescence polarization (FP) assay}

Fluorescence polarization was measured with a PHERAstar (BMG Labtech, Offenburg, Germany) microplate reader by using $485 \pm 20 \mathrm{~nm}$ and $528 \pm 20 \mathrm{~nm}$ band-pass filters (for excitation and emission, respectively). In direct FP measurements, a dilution series of the 14-3-3 protein was prepared in 96-well plates (96 well skirted pcr plate, 4ti-0740, 4titude, Wotton, UK) in a $20 \mathrm{mM}$ HEPES pH 7.5 buffer containing $150 \mathrm{mM} \mathrm{NaCl}, 0.5 \mathrm{mM}$ TCEP, $0.01 \%$ Tween 20, $50 \mathrm{nM}$ fluorescently-labeled fpB6 peptide and $100 \mu \mathrm{M}$ fusicoccin (FSC), if indicated. The volume of the dilution series was $40 \mu \mathrm{l}$, which was later divided into three technical replicates of $10 \mu \mathrm{l}$ upon transferring to 384-well micro-plates (low binding microplate, 384 well, E18063G5, Greiner Bio-One, Kremsmünster, Austria). In total, polarization of the probe was measured at 8 different protein concentrations (whereas one contained no protein and corresponded to the free peptide). In competitive FP measurements, the same buffer was supplemented with the protein to achieve a complex formation of $60-80 \%$, based on the titration. Then, this mixture was used for creating a dilution series of the unlabeled competitor (i.e. the studied peptides) and the measurement was carried out identically as in the direct experiment. Analysis of FP experiments were carried out using ProFit, an in-house developed, Python-based fitting program ${ }^{73}$. The dissociation constant of the direct and competitive FP experiment was obtained by fitting the measured data with quadratic and competitive equation, respectively ${ }^{73,74}$. $\Delta G$ values were calculated using the formula $\Delta G=-R T^{*} \ln \left(K_{D}\right)$, at $295 \mathrm{~K} . \Delta \Delta G_{a v}$ values were obtained by calculating the average and the standard deviation of all obtained individual $\Delta \Delta G$ values (between different motifs or different proteins), excluding cases when $K_{D}>300 \mu \mathrm{M}$.

\section{Crystallization and structure determination}


Crystallization conditions were screened using commercially available and in-house developed kits (Qiagen, Hampton Research, Emerald Biosystems) by the sitting-drop vapor-diffusion method in 96-well MRC 2-drop plates (SWISSCI, Neuheim, Switzerland), using a Mosquito robot (TTP Labtech, Cambridge, UK) at $4{ }^{\circ} \mathrm{C}$. The optimized condition of the crystals consisted of $19 \%$ polyethylene glycol $4000,0.1 \mathrm{M}$ cacodylate buffered at $\mathrm{pH}$ 5.5. For soaking, crystals were transferred to a mother-liquor solution containing (saturated, partially precipitated) $5 \mathrm{mM}$ fusicoccin and crystals were harvested after an $18 \mathrm{~h}$ incubation period. All crystals were flash-cooled in a cryoprotectant solution containing $20 \%$ glycerol and stored in liquid nitrogen.

X-ray diffraction data were collected at the Synchrotron Swiss Light Source (SLS) (Switzerland) on the X06DA (PXIII) beamline and processed with the program XDS ${ }^{75}$. The crystal structure was solved by molecular replacement with a high-resolution crystal structure of 14-3-3 (PDB ID 2O02) using Phaser ${ }^{76}$ and structure refinement was carried out with PHENIX ${ }^{77}$. TLS refinement was applied during the refinement. The crystallographic parameters and the statistics of data collection and refinement are shown in Table 1.

\section{Acknowledgments}

We would like to thank Prof. Lawrence Banks for the shared plasmids, Prof. Alexey Babakov for the provided fusicoccin preparations and Dr. Yaroslav Faletrov for fusicoccin identity verification. N.N.S. is grateful to the Russian Science Foundation for the grant no. 19-74-10031. We thank the support of the Swiss Light Source synchrotron (P. Scherrer Institute, Villigen, Switzerland) and the help of the beam-scientist at the PXIII beamline. The work was supported by the Ligue contre le cancer (équipe labellisée 2015 to G.T.), the French Infrastructure for Integrated Structural Biology (FRISBI), and Instruct-ERIC. G.G. was supported by the Post-doctorants en France program of the Fondation ARC.

\section{Author contributions}

G.G. purified proteins, carried out FP experiments, performed crystallographic studies, analyzed the data and edited the paper. K.V.T. cloned, purified and characterized proteins. C.K. cloned and purified proteins. P.E. synthesized the peptides. G.T. performed data analysis and data interpretation and edited the paper. N.N.S. contributed to protein purification and crystallographic experiments, supervised the research, analyzed the data and wrote the paper.

\section{Conflict of interests}

The authors declare no conflict of interest.

\section{Data availability}

The refined model and the structure factor amplitudes have been deposited in the PDB with the accession codes 6ZFD and 6ZFG. All other data supporting the findings of this study are available from the corresponding authors upon reasonable request.

772 1. Suarez I, Trave G. Structural Insights in Multifunctional Papillomavirus Oncoproteins.

773 Viruses 10, (2018). 
2. Munoz N, et al. Epidemiologic classification of human papillomavirus types associated with cervical cancer. N Engl J Med 348, 518-527 (2003).

3. Bosch FX, et al. Epidemiology and natural history of human papillomavirus infections and type-specific implications in cervical neoplasia. Vaccine 26 Suppl 10, K1-16 (2008).

4. Bouvard V, et al. A review of human carcinogens--Part B: biological agents. The Lancet Oncology 10, 321-322 (2009).

5. Basukala O, Sarabia-Vega V, Banks L. Human papillomavirus oncoproteins and posttranslational modifications: generating multifunctional hubs for overriding cellular homeostasis. Biological chemistry 401, 585-599 (2020).

6. McBride AA. Oncogenic human papillomaviruses. Philos Trans $R$ Soc Lond B Biol Sci 372, (2017).

7. Poirson J, et al. Mapping the interactome of HPV E6 and E7 oncoproteins with the ubiquitinproteasome system. FEBS J 284, 3171-3201 (2017).

8. Celegato M, et al. A novel small-molecule inhibitor of the human papillomavirus E6-p53 interaction that reactivates p53 function and blocks cancer cells growth. Cancer Lett 470, 115-125 (2020).

9. Kolluru S, Momoh R, Lin L, Mallareddy JR, Krstenansky JL. Identification of potential binding pocket on viral oncoprotein HPV16 E6: a promising anti-cancer target for small molecule drug discovery. BMC molecular and cell biology 20, 30 (2019).

10. Zanier K, et al. The E6AP binding pocket of the HPV16 E6 oncoprotein provides a docking site for a small inhibitory peptide unrelated to E6AP, indicating druggability of E6. PLoS One 9, e112514 (2014).

11. Ramirez J, et al. Targeting the Two Oncogenic Functional Sites of the HPV E6 Oncoprotein with a High-Affinity Bivalent Ligand. Angew Chem Int Ed Engl 54, 7958-7962 (2015).

12. Vande Pol SB, Klingelhutz AJ. Papillomavirus E6 oncoproteins. Virology 445, 115-137 (2013).

13. White EA, Kramer RE, Tan MJ, Hayes SD, Harper JW, Howley PM. Comprehensive analysis of host cellular interactions with human papillomavirus E6 proteins identifies new E6 binding partners and reflects viral diversity. J Virol 86, 13174-13186 (2012).

14. Martinez-Zapien D, et al. Structure of the E6/E6AP/p53 complex required for HPVmediated degradation of p53. Nature 529, 541-545 (2016).

15. Masuda Y, et al. Stepwise multipolyubiquitination of p53 by the E6AP-E6 ubiquitin ligase complex. J Biol Chem 294, 14860-14875 (2019). 
822 16. Vats A, Thatte J, Banks L. Identification of E6AP-independent degradation targets of HPV

17. Songyang Z, et al. Recognition of unique carboxyl-terminal motifs by distinct PDZ domains. Science 275, 73-77 (1997).

18. Boon SS, Tomaic V, Thomas M, Roberts S, Banks L. Cancer-causing human papillomavirus E6 proteins display major differences in the phospho-regulation of their PDZ interactions. $J$ Virol 89, 1579-1586 (2015).

19. Ganti K, et al. The Human Papillomavirus E6 PDZ Binding Motif: From Life Cycle to Malignancy. Viruses 7, 3530-3551 (2015).

20. Delury CP, et al. The role of protein kinase A regulation of the E6 PDZ-binding domain during the differentiation-dependent life cycle of human papillomavirus type 18. J Virol $\mathbf{8 7}$, 9463-9472 (2013).

21. Boon SS, Banks L. High-risk human papillomavirus E6 oncoproteins interact with 14-33zeta in a PDZ binding motif-dependent manner. J Virol 87, 1586-1595 (2013).

22. Thatte J, Massimi P, Thomas M, Boon SS, Banks L. The Human Papillomavirus E6 PDZ Binding Motif Links DNA Damage Response Signaling to E6 Inhibition of p53 Transcriptional Activity. J Virol 92, (2018).

23. Kuhne C, Gardiol D, Guarnaccia C, Amenitsch H, Banks L. Differential regulation of human papillomavirus E6 by protein kinase A: conditional degradation of human discs large protein by oncogenic E6. Oncogene 19, 5884-5891 (2000).

24. Gogl G, et al. Dual Specificity PDZ- and 14-3-3-Binding Motifs: A Structural and Interactomics Study. Structure 28, 747-759 e743 (2020).

25. Aitken A. 14-3-3 proteins: a historic overview. Semin Canc Biol 16, 162-172 (2006).

26. Yang X, et al. Structural basis for protein-protein interactions in the 14-3-3 protein family. Proc Natl Acad Sci U S A 103, 17237-17242 (2006).

27. Muslin AJ, Tanner JW, Allen PM, Shaw AS. Interaction of 14-3-3 with signaling proteins is mediated by the recognition of phosphoserine. Cell 84, 889-897 (1996).

28. Yaffe MB, et al. The structural basis for 14-3-3:phosphopeptide binding specificity. Cell 91, 961-971 (1997).

29. Mackintosh C. Dynamic interactions between 14-3-3 proteins and phosphoproteins regulate diverse cellular processes. Biochem J 381, 329-342 (2004).

30. Ganguly S, Weller J, Ho A, Chemineau P, Malpaux B, Klein D. Melatonin synthesis: 14-33-dependent activation and inhibition of arylalkylamine $\mathrm{N}$-acetyltransferase mediated by phosphoserine-205. Proc Natl Acad Sci U S A 102, 1222-1227 (2005). 
871 31. Paiardini A, et al. The phytotoxin fusicoccin differently regulates 14-3-3 proteins association

32. Obsil T, Obsilova V. Structural basis of 14-3-3 protein functions. Semin Cell Dev Biol 22, 663-672 (2011).

33. Stevers LM, et al. Modulators of 14-3-3 Protein-Protein Interactions. J Med Chem 61, 3755 3778 (2018).

34. Luck K, Charbonnier S, Trave G. The emerging contribution of sequence context to the 881

35. Puntervoll $\mathrm{P}$, et al. ELM server: A new resource for investigating short functional sites in modular eukaryotic proteins. Nucleic Acids Res 31, 3625-3630 (2003).

36. Miller CJ, Turk BE. Homing in: Mechanisms of Substrate Targeting by Protein Kinases. Trends Biochem Sci 43, 380-394 (2018).

37. Ben-Shimon A, Niv MY. Deciphering the Arginine-binding preferences at the substratebinding groove of Ser/Thr kinases by computational surface mapping. PLoS Comput Biol 7, e1002288 (2011).

38. Sarabia-Vega V, Banks L. Acquisition of a phospho-acceptor site enhances HPV E6 PDZbinding motif functional promiscuity. The Journal of general virology, (2019).

39. Abraham RT. Cell cycle checkpoint signaling through the ATM and ATR kinases. Genes Dev 15, 2177-2196 (2001).

40. Espejo AB, et al. PRMT5 C-terminal Phosphorylation Modulates a 14-3-3/PDZ Interaction Switch. J Biol Chem 292, 2255-2265 (2017).

41. Crooks GE, Hon G, Chandonia JM, Brenner SE. WebLogo: a sequence logo generator. Genome research 14, 1188-1190 (2004).

42. Stevers LM, et al. Characterization and small-molecule stabilization of the multisite tandem binding between 14-3-3 and the R domain of CFTR. Proc Natl Acad Sci U S A 113, E11521161 (2016).

43. Manschwetus JT, et al. Binding of the Human 14-3-3 Isoforms to Distinct Sites in the Leucine-Rich Repeat Kinase 2. Front Neurosci 14, 302 (2020).

44. Sluchanko NN, Tugaeva KV, Greive SJ, Antson AA. Chimeric 14-3-3 proteins for unraveling interactions with intrinsically disordered partners. Sci Rep 7, 12014 (2017).

45. Tugaeva KV, Remeeva A, Gushchin I, Cooley RB, Sluchanko NN. Design, expression, purification and crystallization of human 14-3-3zeta protein chimera with phosphopeptide from proapoptotic protein BAD. Protein Expr Purif, 105707 (2020). 
46. Woodcock JM, Murphy J, Stomski FC, Berndt MC, Lopez AF. The dimeric versus monomeric status of 14-3-3zeta is controlled by phosphorylation of Ser58 at the dimer interface. J Biol Chem 278, 36323-36327 (2003).

47. Goldschmidt L, Cooper DR, Derewenda ZS, Eisenberg D. Toward rational protein crystallization: A Web server for the design of crystallizable protein variants. Protein Sci 16, 1569-1576 (2007).

48. Nomine $\mathrm{Y}$, et al. Structural and functional analysis of E6 oncoprotein: insights in the molecular pathways of human papillomavirus-mediated pathogenesis. Mol Cell 21, 665-678 (2006).

49. Zanier K, et al. Structural basis for hijacking of cellular LxxLL motifs by papillomavirus E6 oncoproteins. Science 339, 694-698 (2013).

50. Tugaeva KV, Titterington J, Sotnikov DV, Maksimov EG, Antson AA, Sluchanko NN. Molecular basis for the recognition of steroidogenic acute regulatory protein by the 14-3-3 protein family. FEBS J, 10.1111/febs.15474 (2020).

51. Gogl G, et al. Dynamic control of RSK complexes by phosphoswitch-based regulation. FEBS J 285, 46-71 (2018).

52. Camoni L, Visconti S, Aducci P. The phytotoxin fusicoccin, a selective stabilizer of 14-3-3 interactions? IUBMB Life 65, 513-517 (2013).

53. De Vries-van Leeuwen IJ, et al. Interaction of 14-3-3 proteins with the estrogen receptor alpha F domain provides a drug target interface. Proc Natl Acad Sci U S A 110, 8894-8899 (2013).

54. Wurtele M, Jelich-Ottmann C, Wittinghofer A, Oecking C. Structural view of a fungal toxin acting on a 14-3-3 regulatory complex. EMBO J 22, 987-994 (2003).

55. Bier D, et al. Small-Molecule Stabilization of the 14-3-3/Gab2 Protein-Protein Interaction (PPI) Interface. ChemMedChem 11, 911-918 (2016).

56. Sengupta A, Liriano J, Miller BG, Frederich JH. Analysis of Interactions Stabilized by Fusicoccin A Reveals an Expanded Suite of Potential 14-3-3 Binding Partners. ACS Chem Biol 15, 305-310 (2020).

57. Ohkanda J, et al. Structural Effects of Fusicoccin upon Upregulation of 14-3-3Phospholigand Interaction and Cytotoxic Activity. Chemistry 24, 16066-16071 (2018).

58. Kaplan A, et al. Polypharmacological Perturbation of the 14-3-3 Adaptor Protein Interactome Stimulates Neurite Outgrowth. Cell chemical biology 27, 657-667 e656 (2020).

59. Saponaro A, et al. Fusicoccin Activates KAT1 Channels by Stabilizing Their Interaction with 14-3-3 Proteins. Plant Cell 29, 2570-2580 (2017). 
968 60. Kilisch M, Lytovchenko O, Arakel EC, Bertinetti D, Schwappach B. A dual phosphorylation switch controls 14-3-3-dependent cell surface expression of TASK-1. J Cell Sci 129, 831-

61. Centorrino F, Ballone A, Wolter M, Ottmann C. Biophysical and structural insight into the USP8/14-3-3 interaction. FEBS Lett 592, 1211-1220 (2018).

62. Boston PF, Jackson P, Thompson RJ. Human 14-3-3 protein: radioimmunoassay, tissue distribution, and cerebrospinal fluid levels in patients with neurological disorders. $J$ Neurochem 38, 1475-1482 (1982).

63. Wang M, Herrmann CJ, Simonovic M, Szklarczyk D, von Mering C. Version 4.0 of PaxDb: Protein abundance data, integrated across model organisms, tissues, and cell-lines. Proteomics 15, 3163-3168 (2015).

64. Silhan J, et al. 14-3-3 protein C-terminal stretch occupies ligand binding groove and is displaced by phosphopeptide binding. J Biol Chem 279, 49113-49119 (2004).

65. Truong A, Masters S, Yang H, Fu H. Role of the 14-3-3 C-terminal loop in ligand interaction. Proteins 49, 321-325 (2002).

66. Edwards MR, et al. Henipavirus W Proteins Interact with 14-3-3 To Modulate Host Gene Expression. J Virol 94, (2020).

67. Han SI, et al. Rep68 protein of adeno-associated virus type 2 interacts with 14-3-3 proteins depending on phosphorylation at serine 535. Virology 320, 144-155 (2004).

68. Tang $\mathrm{Y}$, et al. 14-3-3zeta binds to hepatitis $\mathrm{B}$ virus protein $\mathrm{X}$ and maintains its protein stability in hepatocellular carcinoma cells. Cancer medicine 7, 5543-5553 (2018).

69. Gu Y-M, Jin Y-H, Choi J-K, Baek K-H, Yeo C-Y, Lee K-Y. Protein kinase A phosphorylates and regulates dimerization of 14-3-3 epsilon. FEBS Lett 580, 305-310 (2006).

70. Sluchanko NN, Uversky VN. Hidden disorder propensity of the N-terminal segment of universal adapter protein 14-3-3 is manifested in its monomeric form: Novel insights into protein dimerization and multifunctionality. Biochim Biophys Acta 1854, 492-504 (2015).

71. Goldschmidt L, Cooper DR, Derewenda ZS, Eisenberg D. SERp Server. (ed^(eds) (2007).

72. Tugaeva KV, Tsvetkov PO, Sluchanko NN. Bacterial co-expression of human Tau protein with protein kinase A and 14-3-3 for studies of 14-3-3/phospho-Tau interaction. PLoS One 12, $\mathrm{e} 0178933$ (2017).

73. Simon MA, et al. High-throughput competitive fluorescence polarization assay reveals functional redundancy in the S100 protein family. FEBS J 287, 2834-2846 (2020). 
1015 74. Roehrl MH, Wang JY, Wagner G. A general framework for development and data analysis

1016 of competitive high-throughput screens for small-molecule inhibitors of protein-protein

1019 75. Kabsch W. Xds. Acta Crystallogr D Biol Crystallogr 66, 125-132 (2010).

1020

1021

1022

1023

1024

1025

1026

1027

1028

76. McCoy AJ, Grosse-Kunstleve RW, Adams PD, Winn MD, Storoni LC, Read RJ. Phaser crystallographic software. J Appl Crystallogr 40, 658-674 (2007).

77. Adams PD, et al. PHENIX: a comprehensive Python-based system for macromolecular structure solution. Acta Crystallogr D Biol Crystallogr 66, 213-221 (2010). 
1030 Table 1. Crystallographic statistics.

14-3-3̧-18E6 chimera

14-3-3ろ-18E6 chimera + FSC

\begin{tabular}{|c|c|c|}
\hline \multicolumn{3}{|l|}{ Data collection } \\
\hline Wavelength & 1.00 & 1.00 \\
\hline Resolution range & $39.26-1.9(1.95-1.9)$ & $38.05-1.85(1.9-1.85)$ \\
\hline Space group & $P 212121$ & P 212121 \\
\hline Unit cell $(a, b, c, \alpha, \beta, \gamma)$ & $72.35,78.53,90.3,90,90,90$ & $73.23,76.1,88.95,90,90,90$ \\
\hline Total reflections & $547783(37726)$ & $557315(41375)$ \\
\hline Unique reflections & $41285(2986)$ & 41927 (3038) \\
\hline Multiplicity & $13.3(12.6)$ & $13.3(13.6)$ \\
\hline Completeness (\%) & $100(100)$ & $97.1(96.5)$ \\
\hline Mean I/sigma(I) & $13.19(1.39)$ & $12.36(1.40)$ \\
\hline R-meas & $16.6(205)$ & $16.3(216)$ \\
\hline $\mathrm{CC} 1 / 2$ & $99.9(54.7)$ & $99.8(58.4)$ \\
\hline \multicolumn{3}{|l|}{ Refinement } \\
\hline R-work & 0.1764 & 0.1908 \\
\hline R-free & 0.2071 & 0.2192 \\
\hline $\begin{array}{l}\text { Number of non-hydrogen } \\
\text { atoms }\end{array}$ & 4468 & 4589 \\
\hline macromolecules & 4034 & 4004 \\
\hline ligands & 24 & 102 \\
\hline solvent & 410 & 483 \\
\hline Protein residues & 481 & 482 \\
\hline RMS(bonds) & 0.006 & 0.006 \\
\hline RMS(angles) & 0.83 & 0.71 \\
\hline Ramachandran favored (\%) & 99.36 & 98.51 \\
\hline Ramachandran allowed (\%) & 0.64 & 1.49 \\
\hline Ramachandran outliers (\%) & 0 & 0 \\
\hline Rotamer outliers (\%) & 1.65 & 2.13 \\
\hline Clashscore & 6.68 & 3.17 \\
\hline Average B-factor & 33.39 & 29.6 \\
\hline macromolecules & 32.42 & 28.49 \\
\hline ligands & 62.93 & 32.67 \\
\hline solvent & 41.2 & 38.11 \\
\hline Number of TLS groups & 15 & 11 \\
\hline PDB ID & 6ZFD & 6ZFG \\
\hline
\end{tabular}

1031

1032

1033 


\section{Figures}
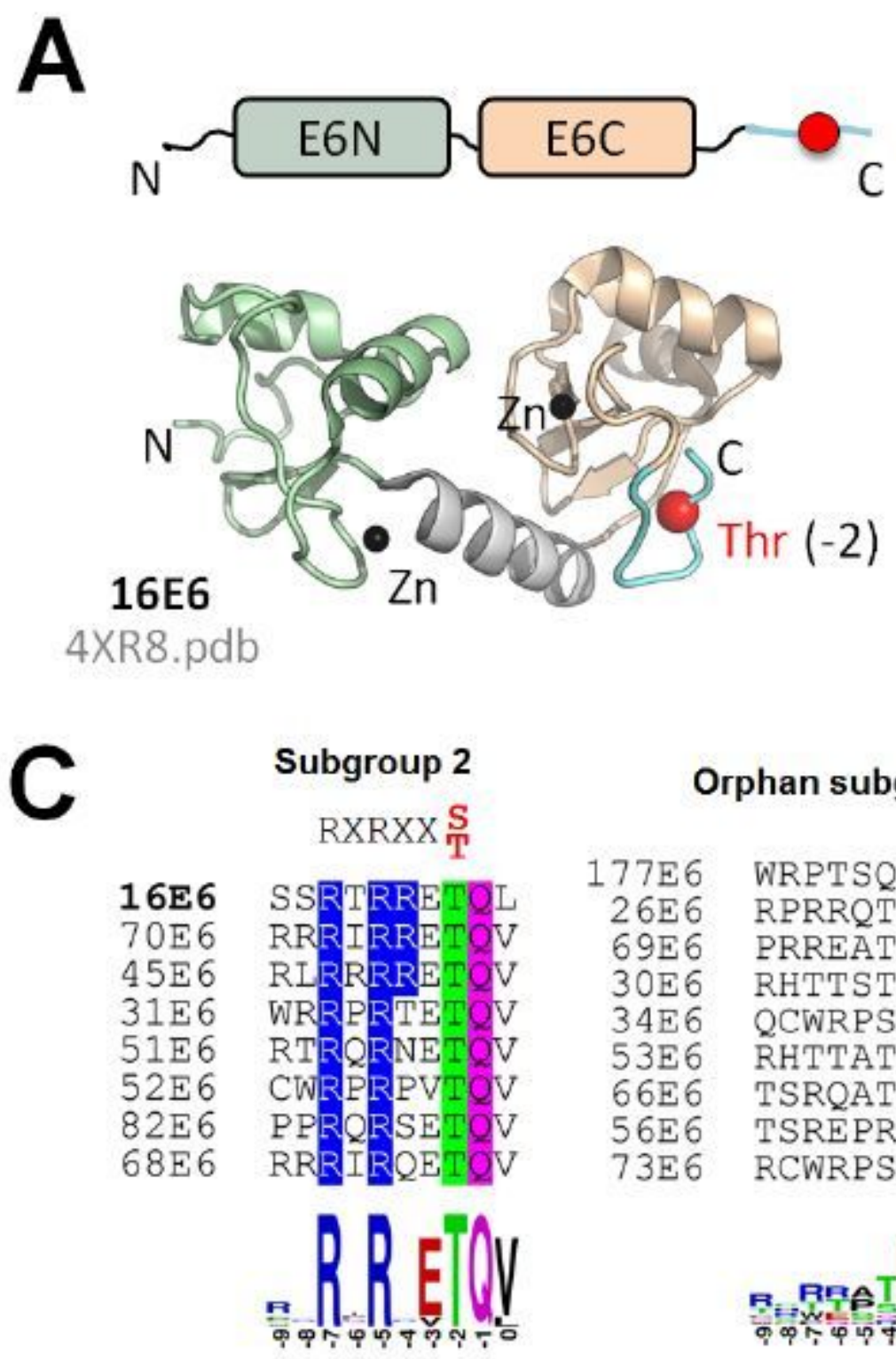

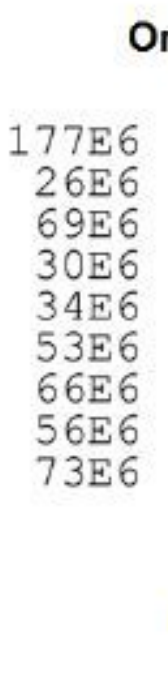

Orphan subgroup
$-9-8-7-6-5-4-3-2-10$

...SSRTRRETQL-COOH 16E6 ...RLQRRRETQV-COOH 18E6 ...WRSRRRETAL-COOH 33E6 ...WKPTRRETEV-COOH 35E6

14-3-3 binding motif III $\ldots \frac{\mathrm{pS}}{\mathrm{pT}} \mathrm{x}_{0-2}-\mathrm{COOH}$ Subgroup 1 RXXS

$18 \mathrm{E} 6$

$97 \mathrm{E} 6$

$39 \mathrm{E} 6$

$85 \mathrm{E} 6$

$58 \mathrm{E} 6$

$67 \mathrm{E} 6$

$35 \mathrm{E} 6$

33E 6

$43 \mathrm{E} 6$

$91 \mathrm{E} 6$

$7 \mathrm{E} 6$

$40 \mathrm{E} 6$

$59 \mathrm{E} 6$

$89 \mathrm{E} 6$

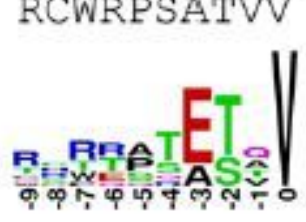

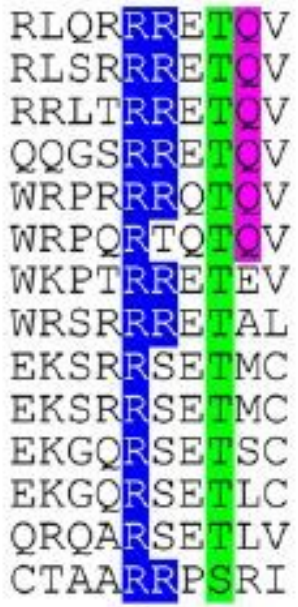

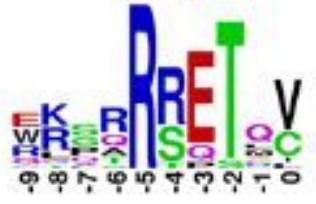

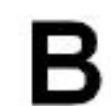

$16 \mathrm{E} 6$
$18 \mathrm{E} 6$
$33 \mathrm{E} 6$
$35 \mathrm{E} 6$

Figure 1

Features of the structure and the C-terminal PDZ-binding motif of E6 proteins. A. The domain organization (top) and three dimensional structure (bottom) of HPV16 E6 (16E6) showing the location of the phosphorylatable PDZ-binding motif (PBM). N-terminal (E6N) and C-terminal (E6C) domains are color coded, structural Zn atoms are in black, PBM is in cyan, phosphorylatable Thr -2 is in red. B. E6 PBMs from high-risk mucosal HPV types overlap with the 14-3-3-binding motif III 30. The positions are numbered above, according to conventional PBM numbering. C. Classification of 31 PBM-containing HPV-E6 proteins based on the correspondence of their C-terminal PBMs to the consensus motifs phosphorylatable by basophilic kinases (subgroups 1 and 2). The third, orphan subgroup comprises PBMs of HPV-E6 proteins whose phosphorylation is less certain. Bold font indicates the E6 PBMs used in this work. Note that many PBMs overlap with recognition motifs (S/TQ) for phosphorylation by DNA 
damage response kinases ATM/ATR (TQ and SQ sites highlighted). Below are shown Weblogo diagrams 41 for the PBMs within each of the three subgroups (positions are numbered according to the PBM convention).

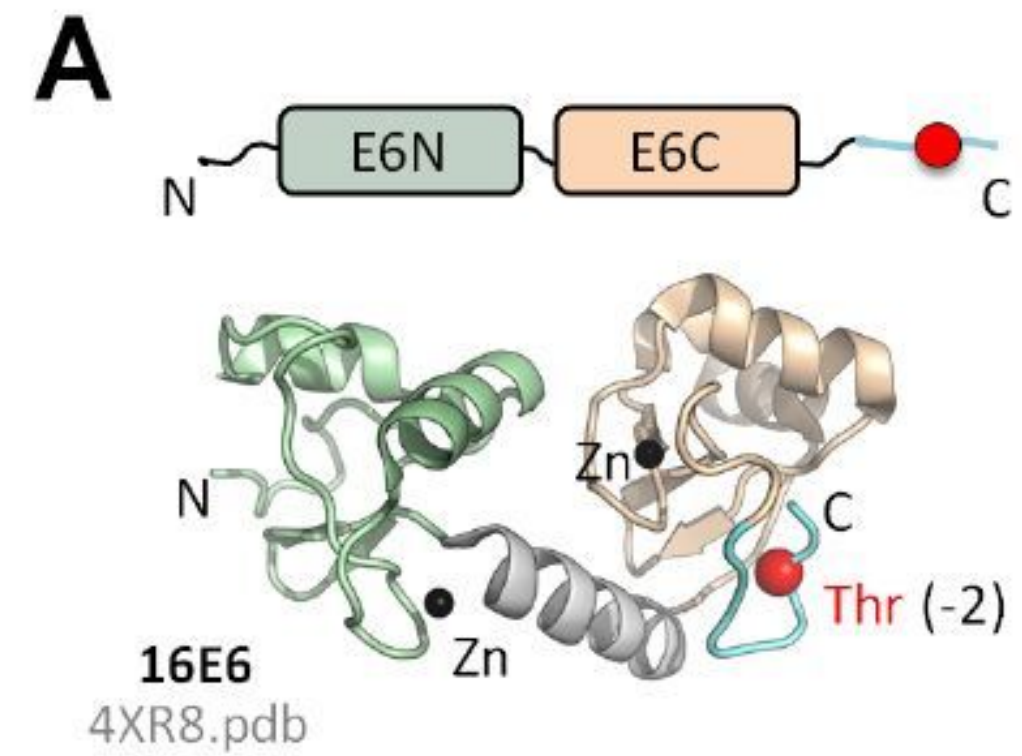

C

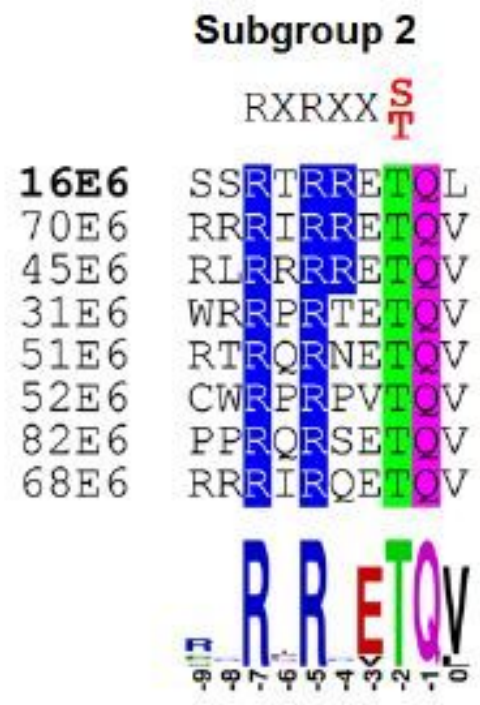

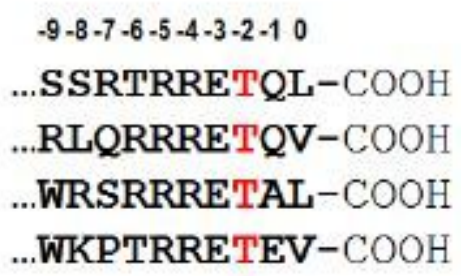

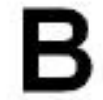

14-3-3 binding motif III

$$
\ldots \frac{\mathrm{pS}}{\mathrm{pT}} \mathrm{x}_{0-2}-\mathrm{COOH}
$$

$16 \mathrm{E} 6$

$18 \mathrm{E} 6$

$33 \mathrm{E} 6$

$35 \mathrm{E} 6$

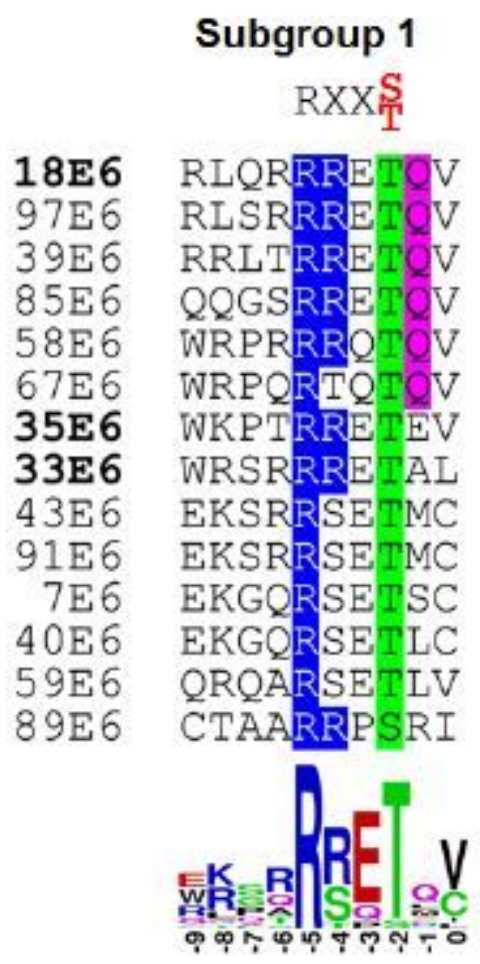

Figure 1

Features of the structure and the C-terminal PDZ-binding motif of E6 proteins. A. The domain organization (top) and three dimensional structure (bottom) of HPV16 E6 (16E6) showing the location of the phosphorylatable PDZ-binding motif (PBM). N-terminal (E6N) and C-terminal (E6C) domains are color coded, structural $\mathrm{Zn}$ atoms are in black, PBM is in cyan, phosphorylatable Thr -2 is in red. B. E6 PBMs from high-risk mucosal HPV types overlap with the 14-3-3-binding motif III 30 . The positions are numbered above, according to conventional PBM numbering. C. Classification of 31 PBM-containing HPV-E6 proteins based on the correspondence of their C-terminal PBMs to the consensus motifs phosphorylatable by basophilic kinases (subgroups 1 and 2). The third, orphan subgroup comprises PBMs of HPV-E6 proteins whose phosphorylation is less certain. Bold font indicates the E6 PBMs used in 
this work. Note that many PBMs overlap with recognition motifs (S/TQ) for phosphorylation by DNA damage response kinases ATM/ATR (TQ and SQ sites highlighted). Below are shown Weblogo diagrams 41 for the PBMs within each of the three subgroups (positions are numbered according to the PBM convention).

\begin{tabular}{|c|c|c|c|c|c|c|c|}
\hline & \multicolumn{7}{|c|}{$K_{D}(\mu M) \pm$ std } \\
\hline & $14-3-3 \gamma$ & $14-3-3 \eta$ & $14-3-3 \zeta$ & $14-3-3 \tau$ & $14-3-3 \beta$ & $14-3-3 \varepsilon$ & 14-3-36 \\
\hline pHPV33E6 & $0.94 \pm 0.25$ & $1.98 \pm 0.15$ & $2.72 \pm 0.39$ & $3.52 \pm 0.28$ & $4.05 \pm 0.65$ & $10.3 \pm 2.39$ & $6.77 \pm 1.13$ \\
\hline pHPV18E6 & $11.1 \pm 0.6$ & $23.6 \pm 6.2$ & $22.4 \pm 1.3$ & $37.8 \pm 6.0$ & $42.2 \pm 6.3$ & $101 \pm 20$ & $139 \pm 24$ \\
\hline pHPV16E6 & $37.2 \pm 3.0$ & $80.5 \pm 16.9$ & $73.6 \pm 19.1$ & $144 \pm 28$ & $159 \pm 45$ & $>300$ & $>300$ \\
\hline pHPV35E6 & $125 \pm 6$ & $163 \pm 19$ & $191 \pm 18$ & $233 \pm 14$ & $>300$ & $>300$ & $>300$ \\
\hline pHPV35E6 $6_{T-6 R}$ & $71.2 \pm 6.7$ & $85.0 \pm 20.6$ & $139 \pm 21$ & $194 \pm 37$ & $179 \pm 29$ & $270 \pm 20$ & $>300$ \\
\hline pHPV35E6 $6_{E-1 A}$ & $9.31 \pm 0.72$ & $12.4 \pm 1.5$ & $17.6 \pm 3.6$ & $21.5 \pm 1.2$ & $20.2 \pm 1.4$ & $31.9 \pm 4.2$ & $44.1 \pm 13.3$ \\
\hline pHPV35E6 6 T-6R, E-1A & $3.27 \pm 0.16$ & $4.77 \pm 0.53$ & $7.32 \pm 0.42$ & $5.84 \pm 0.43$ & $7.06 \pm 0.51$ & $13.0 \pm 2.0$ & $18.9 \pm 1.9$ \\
\hline RSK1_-1P & $0.31 \pm 0.03$ & $0.46 \pm 0.06$ & $0.96 \pm 0.10$ & $0.78 \pm 0.13$ & $0.74 \pm 0.04$ & $1.58 \pm 0.21$ & $1.71 \pm 0.23$ \\
\hline RSK1_-2P & $0.20 \pm 0.01$ & $0.66 \pm 0.05$ & $1.54 \pm 0.09$ & $0.45 \pm 0.08$ & $0.96 \pm 0.03$ & $1.22 \pm 0.16$ & $3.57 \pm 0.32$ \\
\hline
\end{tabular}

B

pHPV33E6 pHPV18E6 pHPV16E6 pHPV35E6

\section{\begin{tabular}{l|l|l|l|l|l|l|}
$14-3-3 \gamma$ & $14-3-3 \eta$ & $14-3-3 \zeta$ & $14-3-3 \tau$ & $14-3-3 \beta$ & $14-3-3 \varepsilon$ & $14-3-3 \sigma$
\end{tabular}}

\section{$\Delta \Delta \mathrm{G}_{\mathrm{av}}=-0.71 \pm 1.19 \quad-1.50 \pm 0.63 \quad-0.34 \pm 0.66 \quad-0.14 \pm 1.39 \quad-0.87 \pm 0.78 \quad-1.35 \pm 0.82 \quad(\mathrm{~kJ} / \mathrm{mol})$ $14-3-3 \sigma<14-3-3 \varepsilon<14-3-3 \beta<14-3-3 \tau<14-3-3 \zeta<14-3-3 \eta<14-3-3 \gamma$ $\Delta \Delta \mathrm{G}_{\mathrm{av}}=\quad-2.06 \pm 0.67 \quad-3.09 \pm 0.15 \quad-5.98 \pm 0.65 \quad(\mathrm{~kJ} / \mathrm{mol})$ HPV35E6 < HPV16E6 < HPV18E6 < HPV33E6}

Figure 2

Summary of affinity measurements carried out in this study. A. Affinities of four selected HPV-E6 phospho-PBMs, p35E6 mutants and RSK1 phosphopeptides towards the seven human 14-3-3 isoforms as determined by fluorescence polarization using FITC-labeled HSPB6 phosphopeptide as a tracer. Apparent KD values determined from competitive FP experiments are presented. $B$. The heatmap representation of the data on panel A showing the affinity trends in the interaction profiles between 14-3-3 isoforms and four HPV-E6 phospho-PBMs from weakest (white) to strongest (red). C. Averaged $\Delta \Delta G$ values between 14-3-3 isoforms or E6 phospho-PBM pairs, calculated based on their observed order of binding affinities (from weakest to strongest). Individual KD values from Supplementary Fig. 1 were first converted into $\Delta \mathrm{G}$ values (at $\mathrm{T}=295 \mathrm{~K}$; excluding cases when $\mathrm{KD}>300 \mu \mathrm{M}$ ) and average $\Delta \Delta \mathrm{G}$ values $(\Delta \Delta \mathrm{Gav})$ were calculated between the indicated motifs/isoforms. 


\begin{tabular}{|l|c|c|c|c|c|c|c|}
\cline { 2 - 9 } \multicolumn{2}{c|}{} & \multicolumn{7}{c|}{$K_{\mathrm{D}}(\boldsymbol{\mu M}) \pm \mathbf{s t d}$} \\
\cline { 2 - 9 } & $\mathbf{1 4 - 3 - 3 \gamma}$ & $\mathbf{1 4 - 3 - 3 \eta}$ & $\mathbf{1 4 - 3 - 3}$ & $\mathbf{1 4 - 3 - 3 \tau}$ & $\mathbf{1 4 - 3 - 3 \beta}$ & $\mathbf{1 4 - 3 - 3 \varepsilon}$ & $\mathbf{1 4 - 3 - 3 \sigma}$ \\
\hline pHPV33E6 & $0.94 \pm 0.25$ & $1.98 \pm 0.15$ & $2.72 \pm 0.39$ & $3.52 \pm 0.28$ & $4.05 \pm 0.65$ & $10.3 \pm 2.39$ & $6.77 \pm 1.13$ \\
\hline pHPV18E6 & $11.1 \pm 0.6$ & $23.6 \pm 6.2$ & $22.4 \pm 1.3$ & $37.8 \pm 6.0$ & $42.2 \pm 6.3$ & $101 \pm 20$ & $139 \pm 24$ \\
\hline pHPV16E6 & $37.2 \pm 3.0$ & $80.5 \pm 16.9$ & $73.6 \pm 19.1$ & $144 \pm 28$ & $159 \pm 45$ & $>300$ & $>300$ \\
\hline pHPV35E6 & $125 \pm 6$ & $163 \pm 19$ & $191 \pm 18$ & $233 \pm 14$ & $>300$ & $>300$ & $>300$ \\
\hline pHPV35E6 & $71.2 \pm 6.7$ & $85.0 \pm 20.6$ & $139 \pm 21$ & $194 \pm 37$ & $179 \pm 29$ & $270 \pm 20$ & $>300$ \\
\hline pHPV35E6 & $9.31 \pm 0.72$ & $12.4 \pm 1.5$ & $17.6 \pm 3.6$ & $21.5 \pm 1.2$ & $20.2 \pm 1.4$ & $31.9 \pm 4.2$ & $44.1 \pm 13.3$ \\
\hline pHPV35E6 & $3.27 \pm 0.16$ & $4.77 \pm 0.53$ & $7.32 \pm 0.42$ & $5.84 \pm 0.43$ & $7.06 \pm 0.51$ & $13.0 \pm 2.0$ & $18.9 \pm 1.9$ \\
\hline RSK1_-1P & $0.31 \pm 0.03$ & $0.46 \pm 0.06$ & $0.96 \pm 0.10$ & $0.78 \pm 0.13$ & $0.74 \pm 0.04$ & $1.58 \pm 0.21$ & $1.71 \pm 0.23$ \\
\hline RSK1_-2P & $0.20 \pm 0.01$ & $0.66 \pm 0.05$ & $1.54 \pm 0.09$ & $0.45 \pm 0.08$ & $0.96 \pm 0.03$ & $1.22 \pm 0.16$ & $3.57 \pm 0.32$ \\
\hline
\end{tabular}

B

\section{\begin{tabular}{l|l|l|l|l|l|l|}
$14-3-3 \gamma$ & $14-3-3 \eta$ & $14-3-3 \zeta$ & $14-3-3 \tau$ & $14-3-3 \beta$ & $14-3-3 \varepsilon$ & $14-3-3 \sigma$
\end{tabular}}

\begin{tabular}{|l|}
\hline PHPV33E6 \\
\hline PHPV18E6 \\
\hline PHPV16E6 \\
\hline PHPV35E6
\end{tabular}

$\Delta \Delta \mathrm{G}_{\mathrm{av}}=\quad-0.71 \pm 1.19 \quad-1.50 \pm 0.63 \quad-0.34 \pm 0.66 \quad-0.14 \pm 1.39 \quad-0.87 \pm 0.78 \quad-1.35 \pm 0.82 \quad(\mathrm{~kJ} / \mathrm{mol})$ $14-3-3 \sigma<14-3-3 \varepsilon<14-3-3 \beta<14-3-3 \tau<14-3-3 \zeta<14-3-3 \eta<14-3-3 \gamma$ $\Delta \Delta \mathrm{G}_{\mathrm{av}}=\quad-2.06 \pm 0.67 \quad-3.09 \pm 0.15 \quad-5.98 \pm 0.65 \quad(\mathrm{~kJ} / \mathrm{mol})$ HPV35E6 < HPV16E6 < HPV18E6 < HPV33E6

\section{Figure 2}

Summary of affinity measurements carried out in this study. A. Affinities of four selected HPV-E6 phospho-PBMs, p35E6 mutants and RSK1 phosphopeptides towards the seven human 14-3-3 isoforms as determined by fluorescence polarization using FITC-labeled HSPB6 phosphopeptide as a tracer. Apparent KD values determined from competitive FP experiments are presented. B. The heatmap representation of the data on panel A showing the affinity trends in the interaction profiles between 14-3-3 isoforms and four HPV-E6 phospho-PBMs from weakest (white) to strongest (red). C. Averaged $\Delta \Delta G$ values between 14-3-3 isoforms or E6 phospho-PBM pairs, calculated based on their observed order of binding affinities (from weakest to strongest). Individual KD values from Supplementary Fig. 1 were first converted into $\Delta \mathrm{G}$ values (at $\mathrm{T}=295 \mathrm{~K}$; excluding cases when $\mathrm{KD}>300 \mu \mathrm{M}$ ) and average $\Delta \Delta \mathrm{G}$ values $(\Delta \Delta \mathrm{Gav})$ were calculated between the indicated motifs/isoforms. 


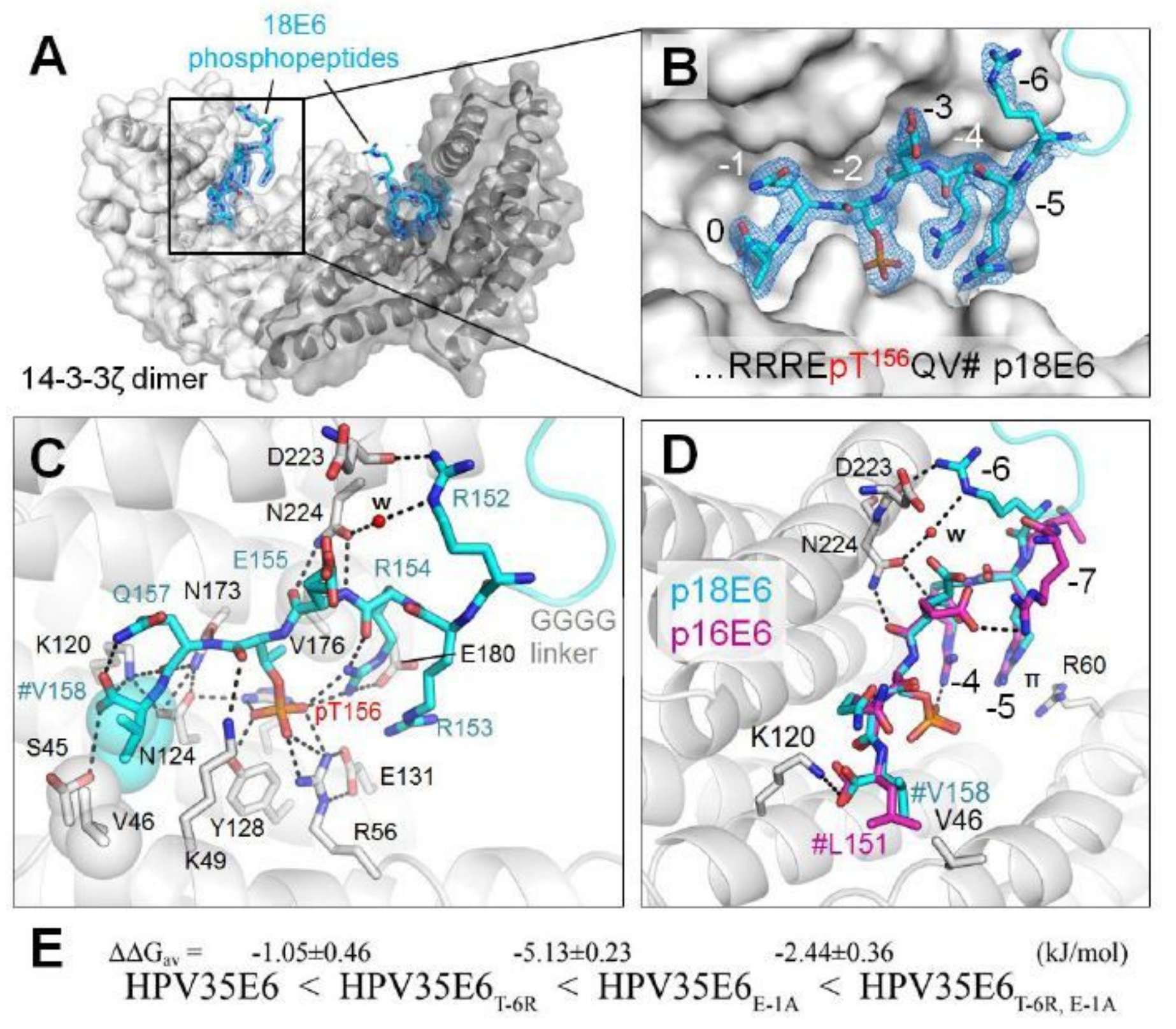

Figure 3

Molecular interface between $14-3-3 \zeta$ and phosphorylated 18 E6 PBM at a $1.9 \AA$ resolution. A. An overall view on the 14-3-3弓 dimer (subunits are in tints of grey) with two bound 18E6 phosphopeptides (cyan sticks). B. A magnified view on one of the amphipathic grooves of 14-3-3 3 showing the conformation of the $18 \mathrm{E} 6$ phosphopeptide and the corresponding $2 \mathrm{Fo}-\mathrm{Fc}$ electron density maps contoured at $1 \sigma$. Positions are numbered according to the PBM convention. C. Polar contacts (dashed lines) and hydrophobic interactions (semitransparent spheres) stabilizing the bound 18E6 peptide conformation. D. An overlay of the two 14-3-3 bound phosphopeptides from 16E6 (6TWZ.pdb) and 18E6 (this work) showing the similarity of the conformation. \# denotes the C-terminus $(-\mathrm{COOH}) . w$ - the water molecule, $\pi-\pi$-stacking interaction. Important positions are numbered according to the PBM convention. E. Averaged $\Delta \Delta \mathrm{G}$ values between 14-3-3 isoforms or 35E6 phospho-PBM pairs, calculated based on their observed order of binding affinities (from weakest to strongest). Individual KD values from Supplementary Fig. 1 were first 
converted into $\Delta \mathrm{G}$ values (at $\mathrm{T}=295 \mathrm{~K}$; excluding cases when $\mathrm{KD}>300 \mu \mathrm{M}$ ) and average $\Delta \Delta \mathrm{G}$ values $(\Delta \Delta \mathrm{Gav})$ were calculated between the indicated motifs/isoforms.

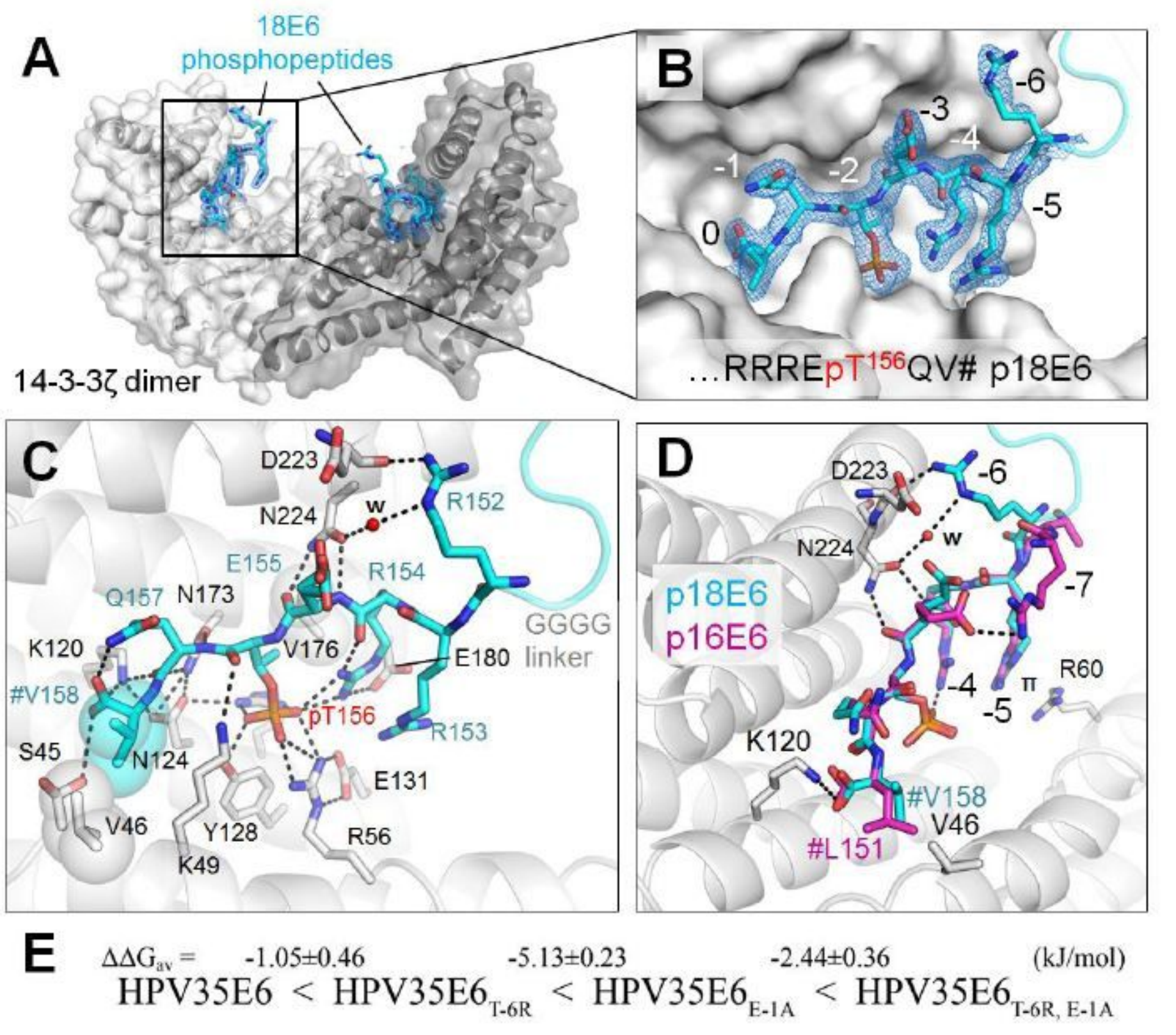

\section{Figure 3}

Molecular interface between $14-3-3 \zeta$ and phosphorylated 18E6 PBM at a $1.9 \AA$ resolution. A. An overall view on the 14-3-3弓 dimer (subunits are in tints of grey) with two bound 18E6 phosphopeptides (cyan sticks). B. A magnified view on one of the amphipathic grooves of 14-3-3 3 showing the conformation of the $18 \mathrm{E} 6$ phosphopeptide and the corresponding $2 \mathrm{Fo}-\mathrm{Fc}$ electron density maps contoured at $1 \sigma$. Positions are numbered according to the PBM convention. C. Polar contacts (dashed lines) and hydrophobic interactions (semitransparent spheres) stabilizing the bound 18E6 peptide conformation. D. An overlay of the two 14-3-3 bound phosphopeptides from 16E6 (6TWZ.pdb) and 18E6 (this work) showing the similarity of the conformation. \# denotes the C-terminus $(-\mathrm{COOH}) . w-$ the water molecule, $\pi-\pi$-stacking interaction. Important positions are numbered according to the PBM convention. E. Averaged $\Delta \Delta \mathrm{G}$ values 
between 14-3-3 isoforms or 35E6 phospho-PBM pairs, calculated based on their observed order of binding affinities (from weakest to strongest). Individual KD values from Supplementary Fig. 1 were first converted into $\Delta \mathrm{G}$ values (at $\mathrm{T}=295 \mathrm{~K}$; excluding cases when $\mathrm{KD}>300 \mu \mathrm{M}$ ) and average $\Delta \Delta \mathrm{G}$ values $(\Delta \Delta \mathrm{Gav})$ were calculated between the indicated motifs/isoforms.
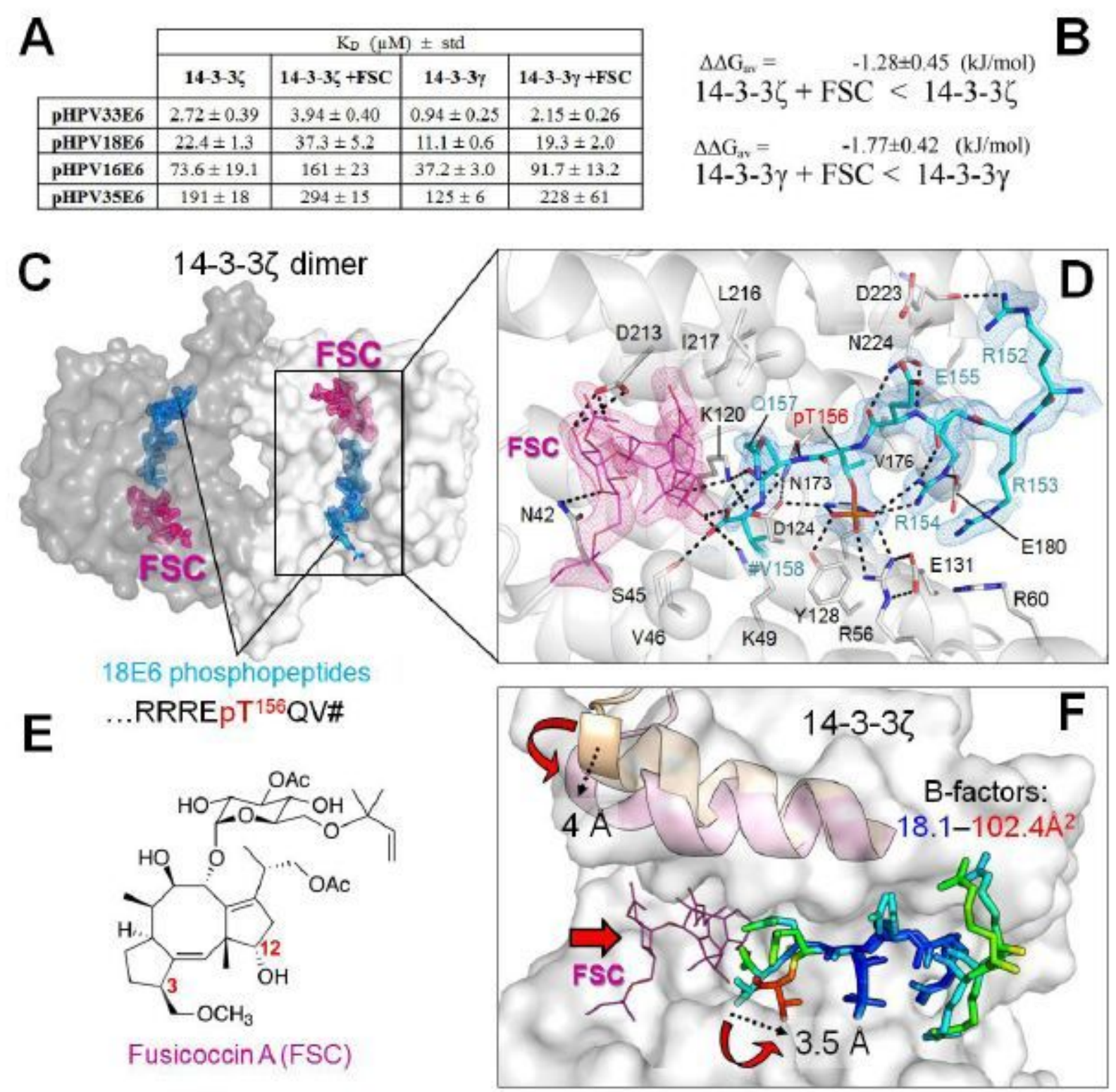

\section{Figure 4}

The destabilizing effect of FSC on the 14-3-3ろ/18E6 PBM interaction. A. Affinities of four selected HPV-E6 phospho-PBMs towards human 14-3-3Z and 14-3-3y in the absence and in the presence of FSC as determined by fluorescence polarization using FITC-labeled HSPB6 phosphopeptide as a tracer. Apparent KD values determined from competitive FP experiments are presented. The binding curves are shown in Supplementary Fig. 1. B. Averaged $\Delta \Delta G$ values between 14-3-3-E6 phospho-PBM pairs in the absence or in the presence of FSC, calculated based on their observed order of binding affinities (from weakest to strongest). Individual KD values from Supplementary Fig. 1 were first converted into $\Delta G$ values (at $T=295$ $\mathrm{K}$; excluding cases when $\mathrm{KD}>300 \mu \mathrm{M})$ and average $\Delta \Delta \mathrm{G}$ values $(\Delta \Delta \mathrm{Gav})$ were calculated between the 
indicated motifs/isoforms. C. An overall view on the ternary complex between 14-3-3ろ (subunits are shown by surface using two tints of grey), 18E6 phosphopeptide (cyan sticks) and FSC (pink sticks). FSC was soaked into the 14-3-3弓-18E6 chimera crystals. 2Fo-Fc electron density maps contoured at $1 \sigma$ show are shown for the phosphopeptide and FSC only. D. A closeup view showing polar contacts (dashed lines) and hydrophobic interactions (semitransparent spheres) positioning the $18 \mathrm{E} 6$ phosphopeptide (cyan sticks) and FSC (thin pink sticks) in the amphipathic groove of the 14-3-3Z subunit (semitransparent light grey ribbon). 2Fo-Fc electron density maps contoured at $1 \sigma$ are shown for the peptide and FSC. \# denotes the C-terminus (- $\mathrm{COOH})$. The GGGG linker is omitted for clarity. E. Chemical formula of FSC showing the positions of the functional groups discussed in the text. F. The effect of FSC binding. Conformational changes upon FSC binding are shown by red arrows reflecting the closure of the C-terminal a-helix of 143-3 and the rotation and translocation of the C-terminal residues in the $18 \mathrm{E} 6$ peptide, accompanied by a significant rise of the local B-factors (are shown as a gradient from blue to red as indicated). The amplitudes of the conformational changes in 14-3-3 and 18E6 peptide are indicated in $\AA$ by dashed arrows.

\begin{tabular}{|c|c|c|c|c|}
\cline { 2 - 5 } \multicolumn{1}{c|}{} & \multicolumn{4}{c|}{$\mathrm{K}_{\mathrm{D}}(\mu \mathrm{M}) \pm$ std } \\
\cline { 2 - 5 } \multicolumn{1}{c|}{} & $\mathbf{1 4 - 3 - 3 5}$ & $\mathbf{1 4 - 3 - 3} ;+\mathbf{F S C}$ & $\mathbf{1 4 - 3 - 3} \boldsymbol{\gamma}$ & $\mathbf{1 4 - 3 - 3 \gamma + F S C}$ \\
\hline pHPV33E6 & $2.72 \pm 0.39$ & $3.94 \pm 0.40$ & $0.94 \pm 0.25$ & $2.15 \pm 0.26$ \\
\hline pHPV18E6 & $22.4 \pm 1.3$ & $37.3 \pm 5.2$ & $11.1 \pm 0.6$ & $19.3 \pm 2.0$ \\
\hline pHPV16E6 & $73.6 \pm 19.1$ & $161 \pm 23$ & $37.2 \pm 3.0$ & $91.7 \pm 13.2$ \\
\hline pHPV35E6 & $191 \pm 18$ & $294 \pm 15$ & $125 \pm 6$ & $228 \pm 61$ \\
\hline
\end{tabular}
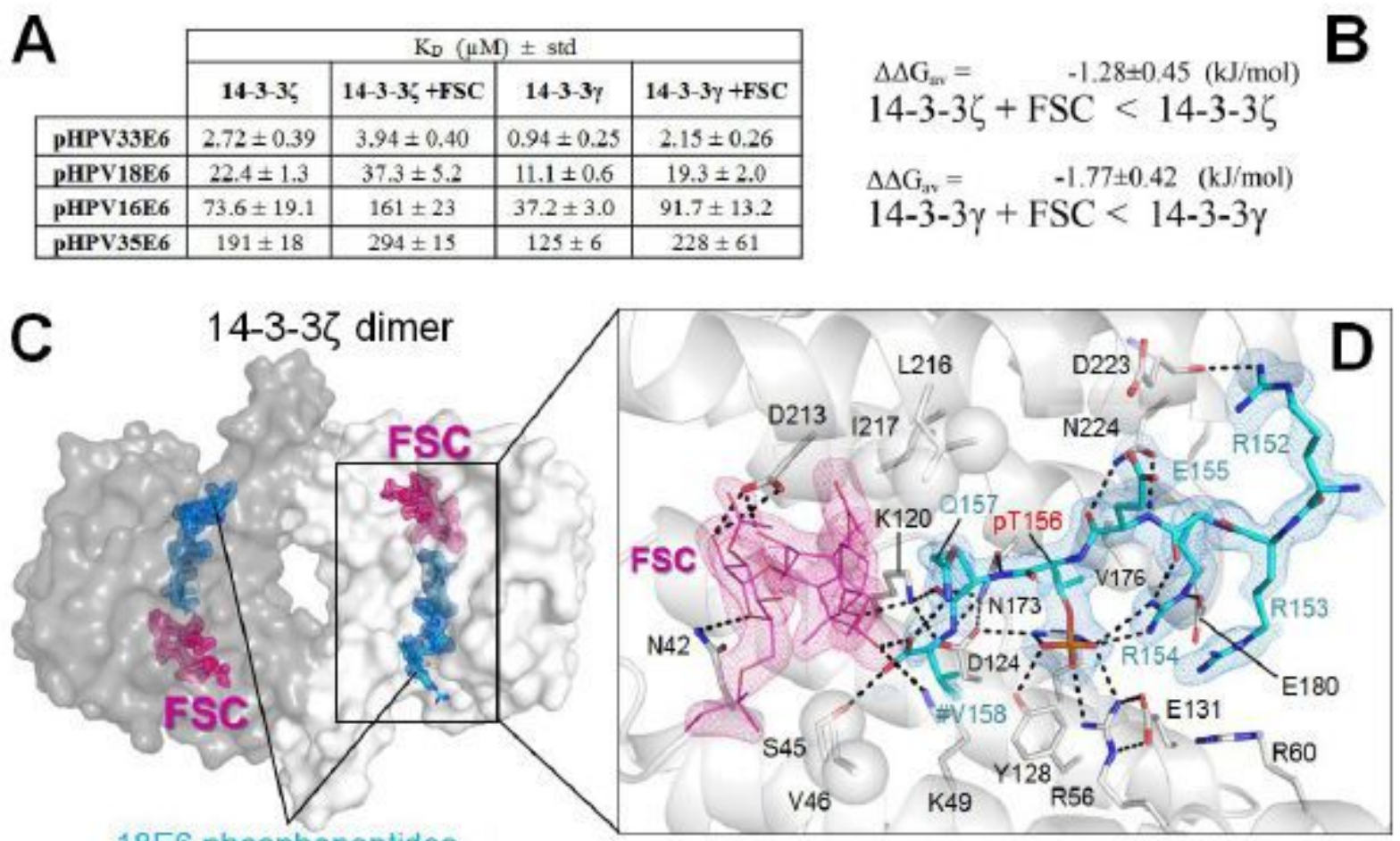

18E6 phosphopeptides

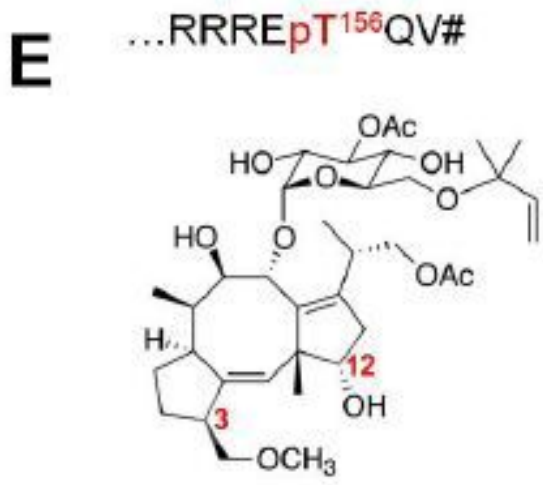

Fusicoccin A (FSC)

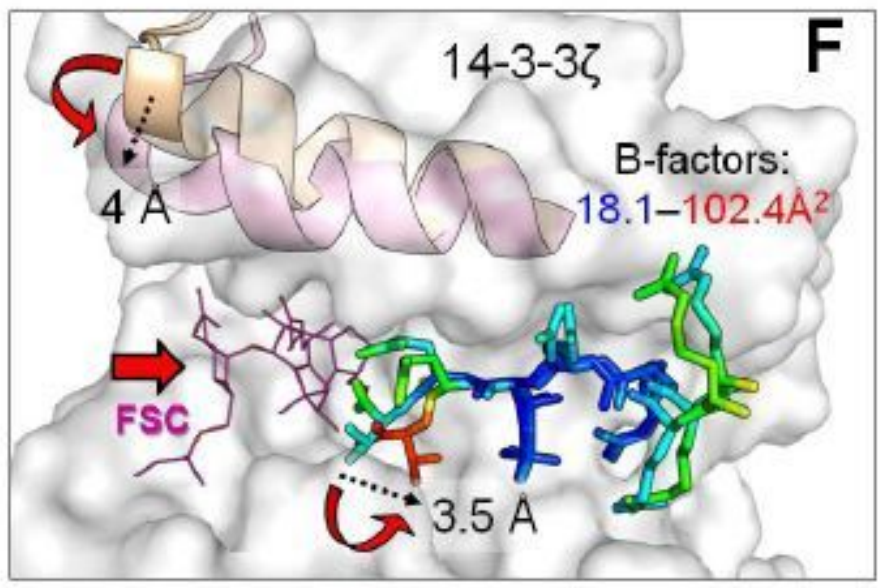




\section{Figure 4}

The destabilizing effect of FSC on the 14-3-3Z/18E6 PBM interaction. A. Affinities of four selected HPV-E6 phospho-PBMs towards human 14-3-3 3 and $14-3-3 y$ in the absence and in the presence of FSC as determined by fluorescence polarization using FITC-labeled HSPB6 phosphopeptide as a tracer. Apparent $\mathrm{KD}$ values determined from competitive FP experiments are presented. The binding curves are shown in Supplementary Fig. 1. B. Averaged $\Delta \Delta G$ values between 14-3-3-E6 phospho-PBM pairs in the absence or in the presence of FSC, calculated based on their observed order of binding affinities (from weakest to strongest). Individual KD values from Supplementary Fig. 1 were first converted into $\Delta G$ values (at $T=295$ $\mathrm{K}$; excluding cases when $\mathrm{KD}>300 \mu \mathrm{M})$ and average $\Delta \Delta \mathrm{G}$ values $(\Delta \Delta \mathrm{Gav})$ were calculated between the indicated motifs/isoforms. C. An overall view on the ternary complex between 14-3-3ろ (subunits are shown by surface using two tints of grey), 18E6 phosphopeptide (cyan sticks) and FSC (pink sticks). FSC was soaked into the 14-3-37-18E6 chimera crystals. 2Fo-Fc electron density maps contoured at $1 \sigma$ show are shown for the phosphopeptide and FSC only. D. A closeup view showing polar contacts (dashed lines) and hydrophobic interactions (semitransparent spheres) positioning the 18E6 phosphopeptide (cyan sticks) and FSC (thin pink sticks) in the amphipathic groove of the 14-3-3Z subunit (semitransparent light grey ribbon). 2Fo-Fc electron density maps contoured at $1 \sigma$ are shown for the peptide and FSC. \# denotes the C-terminus (- $\mathrm{COOH})$. The GGGG linker is omitted for clarity. E. Chemical formula of FSC showing the positions of the functional groups discussed in the text. F. The effect of FSC binding. Conformational changes upon FSC binding are shown by red arrows reflecting the closure of the C-terminal a-helix of 143-3 and the rotation and translocation of the C-terminal residues in the $18 \mathrm{E} 6$ peptide, accompanied by a significant rise of the local B-factors (are shown as a gradient from blue to red as indicated). The amplitudes of the conformational changes in 14-3-3 and 18E6 peptide are indicated in $\AA$ by dashed arrows. 



Figure 5

Comparison of the water-mediated polar contacts formed in the 14-3-3Z/18E6 interface in the absence (A) or in the presence of fusicoccin (FSC) (B). The main residues involved in the interactions are shown by sticks with color coding: 14-3-3 residues are shown in light grey, 18E6 residues are in cyan, phosphogroup of Thr156 is shown by orange sticks. FSC is shown by thin magenta sticks, water molecules affected by FSC binding are shown by lime green, those similar in two structures are black. The Cterminal 18E6 residue (V158) is denoted by \#. Note the significant redistribution of water molecules upon FSC binding. 

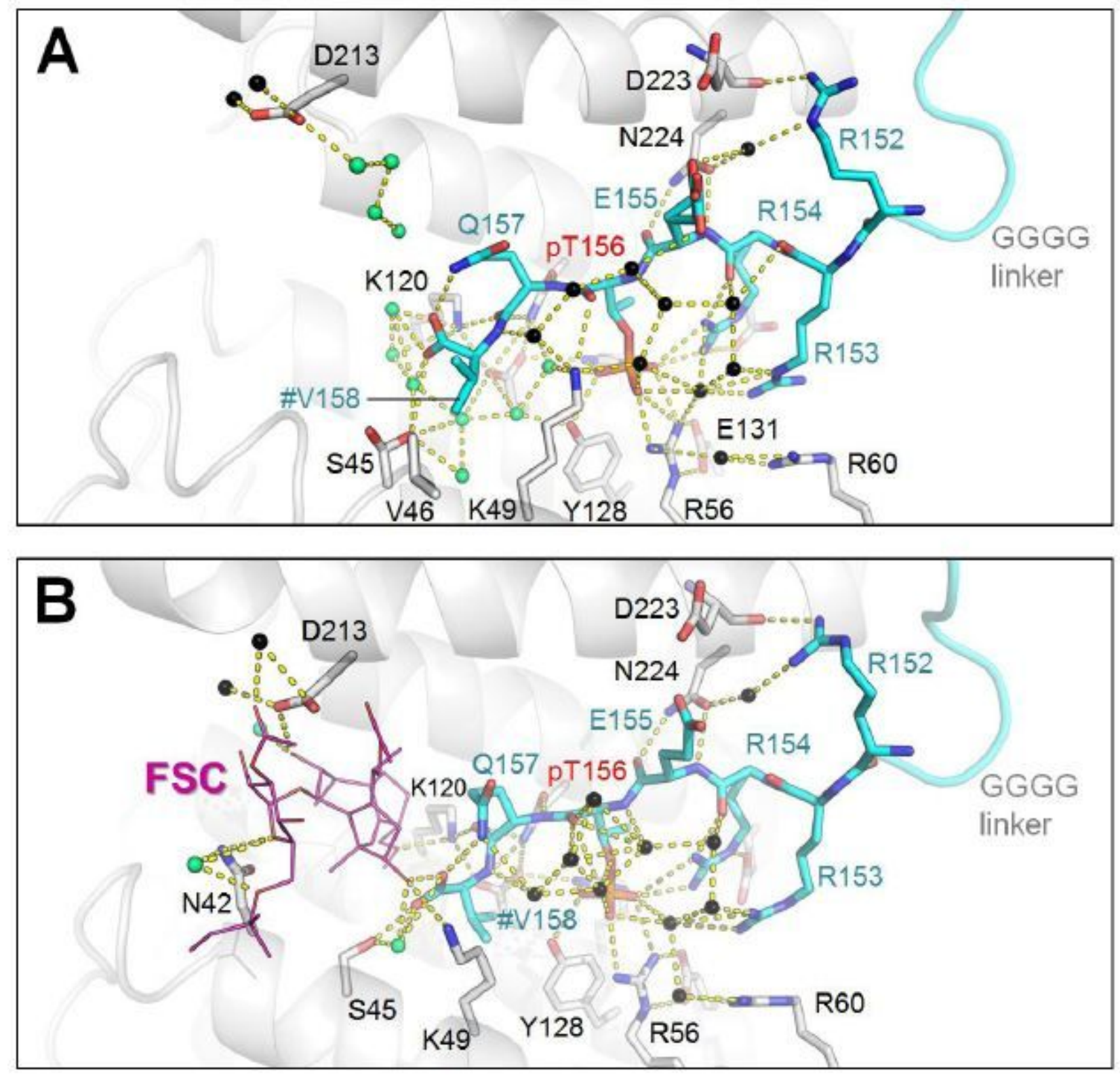

Figure 5

Comparison of the water-mediated polar contacts formed in the 14-3-3Z/18E6 interface in the absence (A) or in the presence of fusicoccin (FSC) (B). The main residues involved in the interactions are shown by sticks with color coding: 14-3-3 residues are shown in light grey, 18E6 residues are in cyan, phosphogroup of Thr156 is shown by orange sticks. FSC is shown by thin magenta sticks, water molecules affected by FSC binding are shown by lime green, those similar in two structures are black. The Cterminal 18E6 residue (V158) is denoted by \#. Note the significant redistribution of water molecules upon FSC binding. 


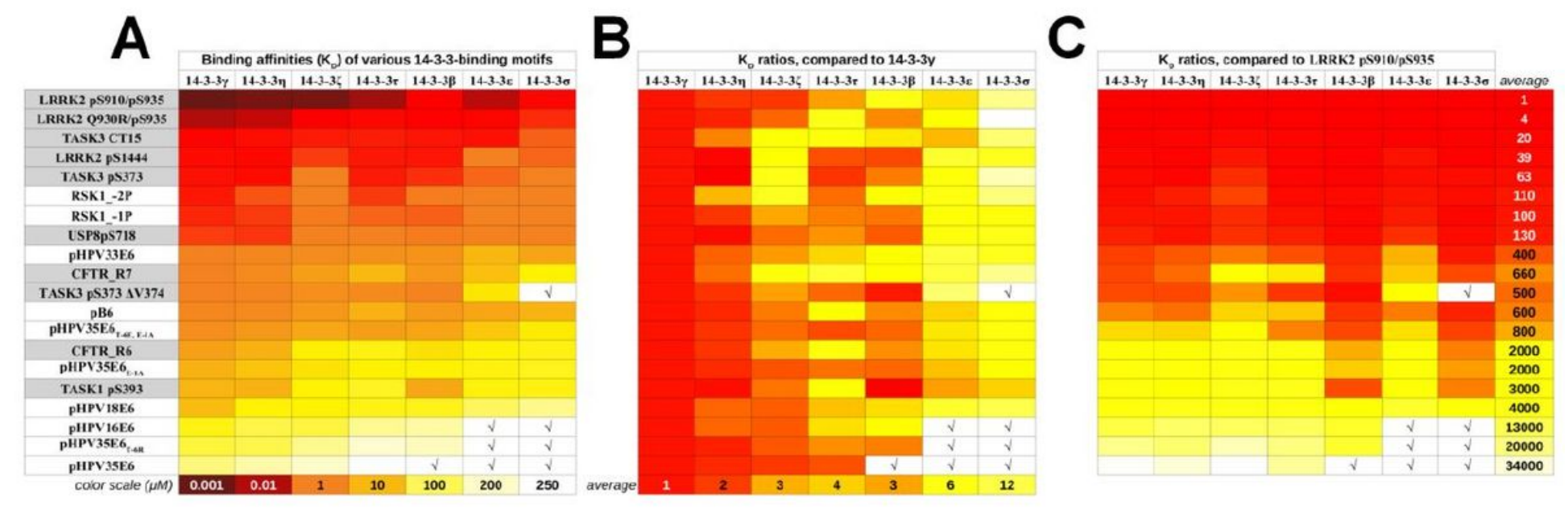

\section{Figure 6}

Compilation of 14-3-3 family-wide affinity measurements of exemplary 14-3-3-binding motifs. A. Affinity maps of 14-3-3 interactions based on experimentally determined dissociation constants against the 14-33ome, as obtained in the current work and in refs $42,43,60,61$. B. When normalized to the strongest partner-binder 14-3-3y, all studied peptides are following very similar affinity trends 586 between the different 14-3-3 isoforms. Between the strongest and the weakest partner-binding 14-3-3 isoform, a 12fold dissociation constant decrease was found on average. $C$. When normalizing to the generally strongest studied 14-3-3-binding motif, the isoform-specific affinity variations for each peptide are compensated to clearly show the average differences between different binding motifs. Between the strongest and the weakest analyzed 14-3-3-binding motif, a 34,000-fold KD decrease was found on average for all 14-3-3 isoforms. Only counting the very similar HPV-E6 peptides sequence-wise, this affinity difference can still be larger than 100 -fold. Therefore, the motif-to-motif affinity differences are expected to be much higher than the 14-3-3 isoform-to-isoform affinity differences. Binding motifs that are analyzed in other studies are highlighted with a grey background $42,43,60,61$. The color scale is either based on affinity values or in $K D$ ratios. $\sqrt{ }$ denotes affinities weaker than the limit of quantitation of the fluorescence polarization assay.
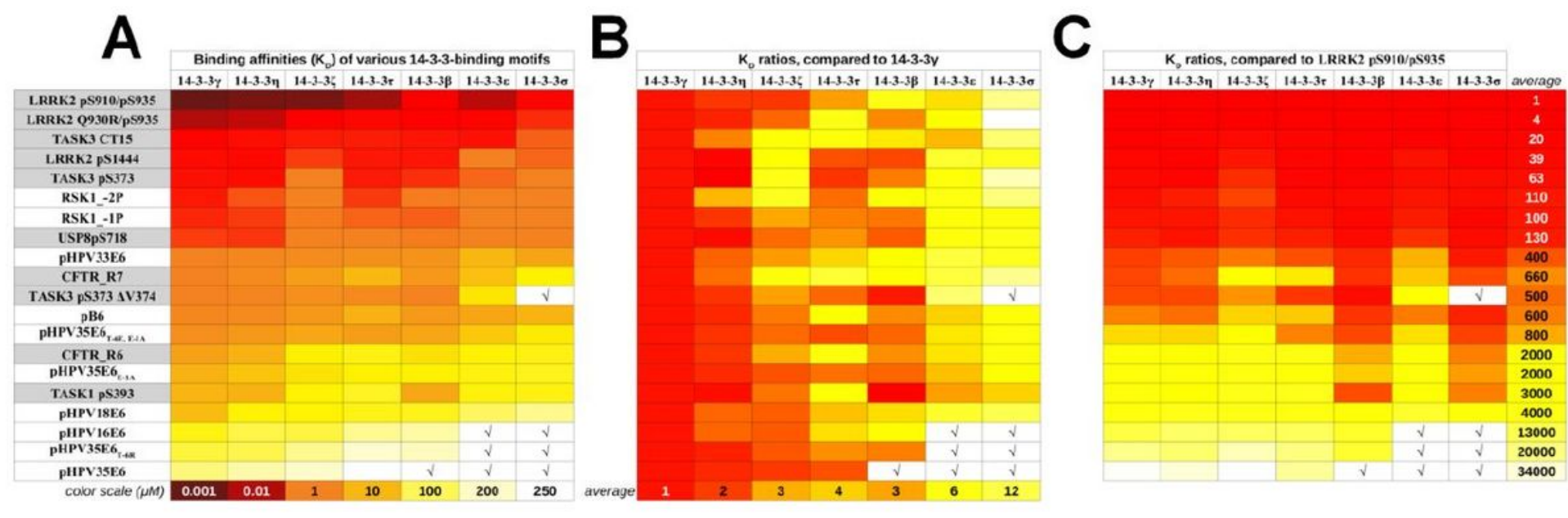


\section{Figure 6}

Compilation of 14-3-3 family-wide affinity measurements of exemplary 14-3-3-binding motifs. A. Affinity maps of 14-3-3 interactions based on experimentally determined dissociation constants against the 14-33ome, as obtained in the current work and in refs $42,43,60,61$. B. When normalized to the strongest partner-binder 14-3-3y, all studied peptides are following very similar affinity trends 586 between the different 14-3-3 isoforms. Between the strongest and the weakest partner-binding 14-3-3 isoform, a 12fold dissociation constant decrease was found on average. C. When normalizing to the generally strongest studied 14-3-3-binding motif, the isoform-specific affinity variations for each peptide are compensated to clearly show the average differences between different binding motifs. Between the strongest and the weakest analyzed 14-3-3-binding motif, a 34,000-fold KD decrease was found on average for all 14-3-3 isoforms. Only counting the very similar HPV-E6 peptides sequence-wise, this affinity difference can still be larger than 100 -fold. Therefore, the motif-to-motif affinity differences are expected to be much higher than the 14-3-3 isoform-to-isoform affinity differences. Binding motifs that are analyzed in other studies are highlighted with a grey background $42,43,60,61$. The color scale is either based on affinity values or in KD ratios. $\sqrt{ }$ denotes affinities weaker than the limit of quantitation of the fluorescence polarization assay.

\section{Supplementary Files}

This is a list of supplementary files associated with this preprint. Click to download.

- D1292109448valreportfullP1.pdf

- D1292109448valreportfullP1.pdf

- D1292109452valreportfullP1.pdf

- D1292109452valreportfullP1.pdf

- 1433E6MSStrasbourgfinalsubmissionSI.pdf

- 1433E6MSStrasbourgfinalsubmissionSI.pdf 